\title{
From SEM images to elastic responses: a stochastic multiscale analysis of UD fiber reinforced composites
}

\author{
Ling $\mathrm{Wu}^{\mathrm{a}, *}$, Chi Nghia Chung ${ }^{\mathrm{b}}$, Zoltan Major ${ }^{\mathrm{b}}$, Laurent Adam ${ }^{\mathrm{c}}$, Ludovic \\ Noels $^{\mathrm{a}}$ \\ ${ }^{a}$ University of Liege, Department of Mechanical and Aerospace Engineering, Computational \\ Es Multiscale Mechanics of Materials, Allée de la découverte 9, B-4000 Liège, Belgium \\ ${ }^{b}$ Johannes Kepler University Linz, Institute of Polymer Product Engineering, \\ Altenbergerstrasse 69, 4040 Linz, Austria \\ ${ }^{c}$ e-Xstream Engineering, Axis Park-Building H, Rue Emile Francqui 9, B-1435 \\ Mont-Saint-Guibert, Belgium
}

\begin{abstract}
In this work, the elastic response of unidirectional fiber (UD) reinforced composites is studied in a stochastic multiscale way. First, the micro-structure of UD carbon fiber reinforced composites is statistically studied based on SEM images of its cross-section and an algorithm to generate numerical micro-structures with an equivalent random distribution of fibers is developed. In particular, based on the images spatial analysis, the empirical statistical descriptors are considered as dependent variables and represented using the copula framework, allowing generating micro-structure realizations used as Stochastic Volume Elements (SVEs). Second, a stochastic scale transition is conducted through the homogenization of SVEs. With a view to the use of the resulting meso-scale random field in structural stochastic analyzes, the homogenization is performed in two steps in order to respect the statistical content from the micro-meter-long SVEs to the millimeter-long structural finite elements. To this end, the computational homogenization is applied in a hierarchy model: i) Micro-structure generator produces Small SVEs (SSVEs) which are homogenized; ii) Big SVEs (BSVEs) are constructed from the SSVEs. Finally, it is shown on simple illustrative examples that the scatter of the (homogenized) stress distribution in a composite ply can be simulated by means of the developed methodology.
\end{abstract}

Keywords: Multiscale, Stochastic, Unidirectional Composites, Stochastic Volume Elements, Micro-structures generator

\footnotetext{
* Corresponding author, Phone: +32436694 53, Fax: +32 43669505

Email addresses: L.Wu@ulg.ac.be (Ling Wu ), Chi_Nghia.Chung@jku.at (Chi Nghia Chung), Zoltan.Major@jku.at (Zoltan Major), laurent.adam@e-xstream.com (Laurent Adam), L.Noels@ulg.ac.be (Ludovic Noels)
} 


\section{Introduction}

Accounting for scatter, variability and uncertainty, as major issues which affect structural and material performance and reliability, has attracted more and more attention from both the scientific and the industrial communities. In different engineering fields, non-deterministic methodologies are being developed to enrich the current modeling and analysis procedures. The sources of variability or uncertainty arise at different structural levels: at the system level in terms of loads and boundary conditions, at the component level in terms of component geometry, but also at the material level in terms of its mechanical properties. This work focuses on the latter aspect.

Uncertainty is an inherent nature of materials, especially for composite materials, in which the uncertainty due to the heterogeneities has a significant impact on the material properties and might influence the response of structures made of that material as well. Stochastic Finite Element Method (SFEM), as an extension of the deterministic Finite Element Method (FEM), can solve static and dynamic stochastic problems involving uncertainties resulting from material properties [1-3]. By using finite elements whose properties are random, SFEM can propagate the uncertainties through the mechanical system and assess the structural stochastic response $[4,5]$. However, in order to obtain a reliable structural analysis by SFEM, a well defined stochastic description of the material properties is required.

A limited number of mechanical tests cannot reliably sample the material properties distributions, since this would require an excessive number of tests. To overcome this issue, the concept of virtual stochastic tests is developed. Although a stochastic model of macro-scale composite material properties can be built from direct macro-scale measurements [6, 7, e.g.], there is a growing interest to account in an explicit way for the uncertainties related to material micro-structure. The stochastic description then relies either on mathematics models or on database, which are characterized by a quantification of the material uncertainties. Indeed, the uncertainty due to the material heterogeneities can be described as the spatial variability of the material properties at the micro-structure level. According to the material systems and material scales of interest, different techniques are applied to assess the micro-structure spatial variability. In the context of composite materials, X-ray microfocus computed tomography (micro-CT) is often used to characterize the Three-Dimensional (3D) spatial geometrical variability of fiber tows $[8,9]$ and defects [10] in the scale of a few millimeters, and 3D Digital Image Correlation (DIC), as a complementary technique, can be used to characterize long-range weave defects in textile composites [11]. For unidirectional (UD) composites, the spatial characterization often focuses on the material cross-sections, and their Two-Dimensional (2D) images can be acquired via the Scanning Electron Microscope (SEM) in scales ranging from a few to hundreds of micrometers $[12,13]$. From the characterized stochastic features, virtual specimen generators are then constructed to create large numbers of replicas of the micro-structure as proposed in $[9,10,14]$ for textile composites, in $[12,15]$ for UD composites and in [16] for particle 
reinforced composites. The replicas serve as virtual specimens in simulations that can reveal the correlation between the micro-structural variations and the material performance.

Theoretically, the size of generated virtual specimens could reach the size of macro-specimens $[9,10]$ or, when performing a structural analysis, the structures can be generated with the spatial geometrical variability. However, direct numerical simulations accounting explicitly for the material structure cannot always be envisioned due to the huge computational cost. Therefore, because of the different involved length scales [17], there is a need to develop framework to up-scale the uncertainties by formulating a computationally efficient stochastic scale transition to bridge the gap between the material scale and the structural scale. Most of the time, the generated virtual specimens serve as Stochastic Volume Elements (SVEs) in a stochastic multiscale analysis. The development of stochastic scale-transition methods is a very recent subject of research, even for linear systems.

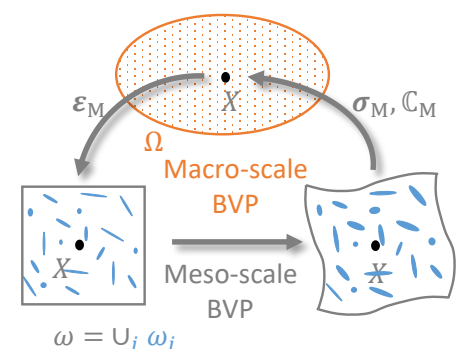

Figure 1: Definition of the homogenization method performed on the meso-scale volume element $\omega$.

Among the existing deterministic multiscale methods, homogenization-based ones were extensively developed, see the reviews $[18,19]$. In such an approach, the macro-scale structure defines a Boundary Value Problem (BVP) which is solved by considering homogenized material properties extracted, at each (macro) material point of interest, from the resolution of a meso-scale BVP, see Fig. 1. This meso-scale BVP is defined on a meso-scale volume which represents the different phases of the material. The resolution of this mesoscale BVP links the macro-, or homogenized-, stress tensor and the macro-, or homogenized-, strain tensor, which correspond to the average values of respectively the local stress tensor and strain tensor on the meso-scale volume element. In case of statistical representativity of the meso-scale problem, the meso-scale volume element is called Representative Volume Element (RVE). In case the meso-scale volume element does not respect the statistical representativity, it is called Statistical or Stochastic Volume Element (SVE) [5]. The so-called computational homogenization developed in [20-24] can be applied on SVEs to define the probability convergence criterion of RVE for masonry [25], for poly-silicon [26] and for composite materials [27]. When combined to a Monte Carlo (MC) resolution of SVEs, the computational homogenization is used to study meso- 
scale properties in a statistical way: e.g. to evaluate in a probabilistic way the parameters of a meso-scale porous constitutive model [28], open foams mesoscale properties [29], the effective properties of random two-phase composites $[27,30,31]$, the meso-scale properties of poly-silicon in the context of elasticity [32] and thermo-elastic damping [33], or to study the scale-dependency of homogenization for random composite materials [34, 35]. Poly-silicon thin structures uncertainties resulting from material and surface roughness can be considered altogether through second-order homogenization on SVEs in [36]. When the spatial correlation is also captured during the stochastic homogenization process, e.g. using a moving-window technique [37], a meso-scale properties random field is defined, which can in turn be used as input of the macro-scale SFEM.

Therefore, when considering the material uncertainties from the properties spatial variability at the micro-scale, the process of stochastic multiscale analyzes can be summarized as i) The assessment of the spatial variability of the micro-structure, to acquire its stochastic description; ii) The construction of SVEs' generator and the homogenization of the SVEs to obtain the stochastic material properties at the meso-scale; and iii) The structural analysis with the stochastic material properties obtained at the meso-scale.

However in order to account for general micro-scale randomness, the resolution of the meso-scale problems during the resolution of the structural-scale analysis can lead to a prohibitive cost when considering probabilistic studies. This has motivated the constructions of meso-scale random fields generators. The meso-scale random field generator can be built based on a mathematical formulation, but it can also be a database driven generator in which the empirical Probability Distributions Functions (PDF) of random variables and discrete correlation functions are used. Meso-scale random field generators can be built using a Karhunen-Loève expansion as in the context of the meso-scale porous constitutive model [38] or of open foams [29], using the maximum entropy principle $[39,40]$ as in the context of polycrystalline materials, or using a spectral representation method [41] combined to a non-Gaussian mapping technique [4244] as in the context of poly-silicon elasticity [32], thermo-elastic damping [33], and plate bending [36], or again in the context of 2-phase composite materials [31]. The generated meso-scale random fields are then used in an efficient way as input of the SFEM to up-scale the micro-scale uncertainties to the structural behaviors.

This work focuses on the stochastic multiscale analysis of UD-fiber reinforced composites. Indeed, the spatial arrangement of fibers in a composite micro-structure is normally not periodic and can have a significant effect on the failure/damage initiation and evolution under certain loading conditions $[13,45,46]$. To illustrate this behavior, Fig. 2 studies the effect of the microstructure characteristics of hexahedral micro-structured UD composites, i.e. the loading orientation $\theta$ with respect to the fiber lattice and the minimum distance between fibers $d_{\min }$, on the response of transversely loaded SVEs. In this model, the fibers follow a transverse isotropic elastic material model, while the matrix follows a damage enhanced elasto-plastic model described in [47], which 


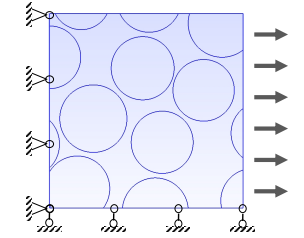

(a)

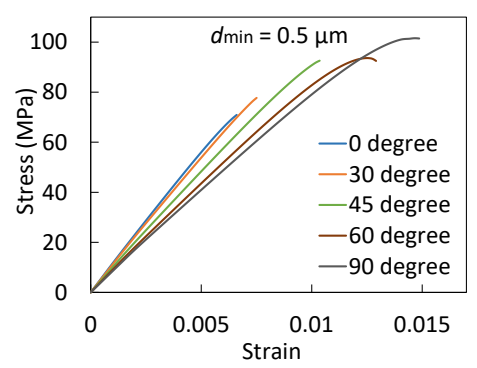

(c)

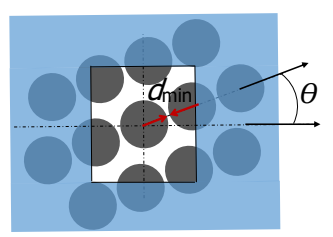

(b)

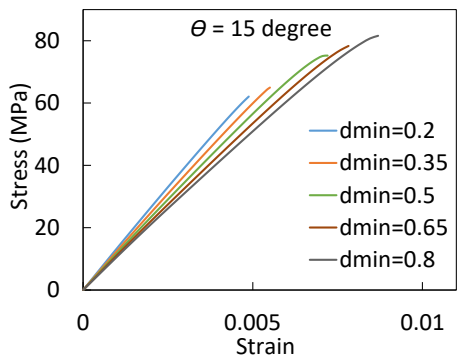

(d)

Figure 2: Study of the loading orientation and of the net minimum distance between fibers effects on the homogenized behavior of hexahedral micro-structured UD composites: (a) Definition of the SVE loading conditions; (b) Definition of the loading orientation $\theta$ and of the minimum net distance between fibers $d_{\min }$; (c) Loading curves for a given $d_{\min }=0.5 \mu \mathrm{m}$; (d) loading curves for a given $\theta=15^{\circ}\left(d_{\min }\right.$ in $\left.\mu \mathrm{m}\right)$.

allows approximating the failure point of the composite material. The material properties of the two phases have been identified in [48]. For a given microstructure parameter kept constant, $d_{\min }=0.5 \mu \mathrm{m}$ in Fig. 2(c) and $\theta=15^{\circ}$ in Fig. 2(d), it can be seen that both the elastic part of the response and the maximum homogenized stress reached upon composite material failure depend on the other parameter, respectively the orientation $\theta$ and the net minimum distance between fibers $d_{\min }$. The purpose of this work is to study in a stochastic multiscale way the effect of UD-fiber micro-structure uncertainties on the composite material elastic behavior. In a future work, the effect of the micro-structure uncertainties on failure will be accounted for, e.g. by defining probabilistic fracture strength from SVE simulations as in $[49,50]$.

As the first addressed aspect of the paper, the micro-structure of UD carbon fiber reinforced composites is statistically studied based on SEM images of its cross-section. A new algorithm to generate SVEs with an equivalent random distribution of fibers is then presented. There exist two main kinds of random fiber generation algorithms: additive and compact. The additive process [12] uses the statistical spatial descriptors of point patterns, such as neighboring fiber distances, to position fibers successively in the target area. Although the distribution of fiber radius is applied in the fiber generating process, it is treated independently from its neighboring fiber distances, which increases the possibility of fiber being rejected because of overlapping. The dependency among the nearest neighbor distances and orientations, such as between the first and second 
nearest neighbor distances, is not considered either. The compact process [15] stirs and compacts the existing fibers iteratively to obtain a random fiber distribution with a target fiber volume fraction. The generated distributions were analyzed using statistical functions and a good agreement was found compared with the completely spatial randomness of a Poisson distribution. However, for a real material the fiber volume fractions of SVEs are uncertain values [27] and the spatial point pattern of fibers does not necessarily follow a Poisson distribution. In this work, based on the spatial analysis of SEM images, the empirical statistical descriptors of nearest neighbor distances and orientations are used in the fiber additive process. Moreover, the observed dependency of the empirical statistical descriptors is also accounted for. The statistical analysis of the generated SVEs shows that the achieved fiber volume fraction is defined by the statistical spatial descriptors of the material micro-structure, which indicates the rationality of using an additive algorithm to produce SVEs.

The second aspect addressed in this work is on improving the computational efficiency of the scale transition from SVEs to structures. In [32] direct MC simulations of micro-electromechanical system realizations are compared to the stochastic multiscale predictions obtained by combining the random field generator and the SFEM. This showed that accounting for the spatial correlation through the meso-scale random field ensures the accuracy of the method if the SVEs have a size comparable to the structural finite elements. However, this requirement is not always possible for composite materials. Although for textile composites, the generated SVEs have a size of millimeters and their homogenized properties can be used directly as meso-scale random fields for structural analyzes [51], for UD-fiber reinforced composites, the characteristic size of SVEs is typically in the scale of the micrometer for computational efficiency. Indeed, the direct FEM analysis on the SVEs of UD composites is rather time consuming in the perspective of non-linear and failure analyzes. Considering the computational efficiency of a statistical analysis, which requires a huge amount of simulations on SVEs, the size of SVEs that can be considered is limited. Therefore, for the stochastic analysis of UD composites and other heterogeneous materials which face the same challenge, the researches are usually limited in analyzing the stochastic responses of SVEs [15, 34, 37]. Although the homogenized properties of SVEs have been used in up-scaling analyzes, the upscale samples were still limited to the micrometers [37]. In a real FEM analysis of composite structures, the finite element sizes are of the millimeter order. This requires the SVEs used to define the meso-scale random fields to have a comparable size. In order to be able to carry out a statistical analysis on big SVEs of hundreds of micrometers in a efficient way, a multi-level homogenization procedure is used in this work. The computational homogenization is applied in a hierarchy model: i) A micro-structure generator produces Small SVEs (SSVEs) which are homogenized computationally; and ii) Big SVEs (BSVEs) are constructed from the SSVEs and the homogenized properties of SSVEs are used as material properties of the BSVEs. During this analysis, two aspects are studied in details to assess the accuracy of the method. On the one hand, the effects of boundary conditions during the computational homogenization are studied, and 
periodic boundary conditions are shown to minimize the error. On the other hand, in some applications the homogenized properties are spatially correlated on distances larger than the SVEs, which requires the use of overlapping SVEs to define the homogenized random field [32, 37]. However, for UD composites the numerical analysis shows that more accurate homogenized results of the BSVEs are provided by using non-overlapping SVEs, which allows considering material properties as random vectors during the stochastic analysis at the structural level.

The paper is organized as follows. In Section 2 SEM images of UD crosssections are statistically characterized through the spatial point pattern description functions. In particular the dependency between spatial parameters is investigated. Section 3 describes the generator developed to produce SVEs, which respect the statistical characteristics experimentally observed. The stochastic homogenization is performed in Section 4, in which the accuracy of the two-level homogenization process is investigated. Finally, simple composite ply stochastic analyzes are conducted in Section 5 to illustrate the applicability of the stochastic multiscale methodology.

\section{Statistical analysis of the micro-structure of the UD-fiber rein- forced epoxy cross section}

The spatial and geometric information of UD-fiber reinforced epoxy crosssection are obtained by SEM. The spatial point pattern description functions of the cross section's micro-structures are then extracted from the SEM images. The statistical analysis of the spatial point pattern is then used to describe the cross-section of UD composites. Moreover, the radii of the fibers, which also possess uncertainty, can be taken into account in a marked point process.

\subsection{Image acquisition}

On the one hand, the resolution of a SEM image is limited by the instrument. On the other hand, the required resolution is governed by the material system. For example, in the case of SEM images with an amplification of $500 \times$, one pixel corresponds to a distance of about $0.22 \mu \mathrm{m}$ and the shape of the fibers' cross-section cannot be captured accurately. Therefore, only SEM images with a high amplification ratio, such as $3000 \times$ or $2000 \times$, can be used to extract the information of fibers' cross section and their relative distances. This means that the point process in a large observation window, which contains at least hundreds of fibers, cannot be obtained accurately from a direct SEM image process. Therefore, based on the assumption of the stationary or homogeneity of the point process, the solution for large observation windows is obtained by the sum of information from several small windows.

\subsection{Image processing}

In this work, 103 SEM images of amplification ratios $3000 \times$ and $2000 \times$ were used. These SEM images of the material system were processed using MATLAB $^{\mathrm{TM}}$ image processing toolbox by successively: 


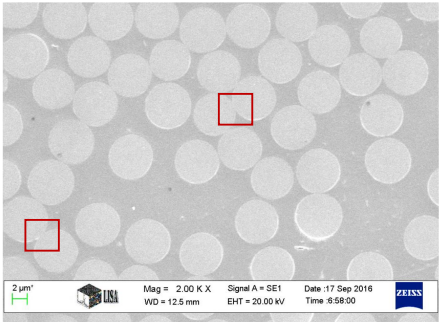

(a) Original

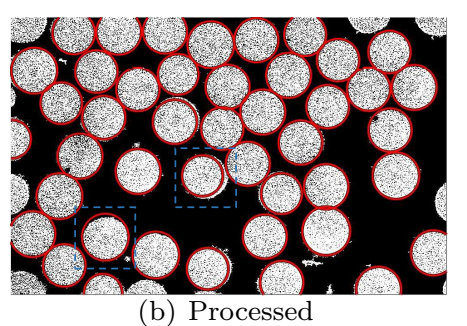

(b) Processed

Figure 3: A SEM image of a polished cross section sample with an amplification ratio of 2000×: (a) Original image, the square represent zero-distance fibers; (b) Processed image with the detected fibers, the dashed squares represent the over/under-estimated fiber circles.

1. Using unsharp masking to sharpen the original SEM images and cropping off the part of images with operation information;

2. Converting the enhanced images into black and white images and removing all connected components (objects) that correspond to less than a given pixels number;

3. Identifying the fibers with a circles detection tool.

Figure 3 gives an example of an original SEM image, Fig. 3(a), and of the processed image with the detected circles, Fig. 3(b).

\subsection{Basic geometric information of fibers' cross sections}

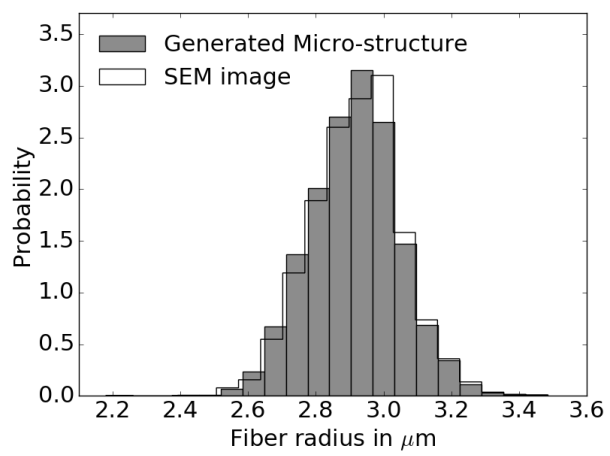

Figure 4: The histograms of fiber radius obtained from SEM images of amplification ratios $3000 \times$ and $2000 \times$ and from the generated micro-structures.

When we use the circles detection function to extract the geometric information of the fibers, because the cross sections of real fibers are not perfectly round, the radii of the fibers might be over or underestimated, as being shown in the dashed blue squares in Fig. 3(b). However, using the SEM images obtained with an amplification ratio of $3000 \times$ or $2000 \times$, we can get more detailed information on the shape of a fiber's cross section. The study of few hundreds 
fibers gives an average fibers' roundness of 1.045 with a standard deviation of $0.011^{1}$. This result proves that the use of circles to represent the fibers' cross section is a reasonable assumption. Moreover, only a few fiber radii are over or underestimated and these over or underestimated ones are easily seen and can be modified manually. Figure 4 shows the histograms of fiber radius $R$ based on the analysis of the SEM images and this result will be used in Section 3 to generate random fiber radii in the Stochastic Volume Elements (SVE).

We need to note that the penetration of neighboring fibers, that could possibly arise during the circle detection process, cannot be permitted, but that a zero-distance between them is acceptable, see the red squares in Fig. 3(a). When a penetration happens between the detected neighboring fibers, a minimum distance, $d_{\min }$, is set at the boundaries of these fibers, and the two fibers radii are reduced to satisfy this minimum distance. This random minimum distance has the highest probability to be zero and its upper limit is defined by the resolution of the images. Since in the images with an amplification ratio of $2000 \times$, one pixel corresponds to a distance of about $0.057 \mu \mathrm{m}$, we assume $d_{\text {min }}$ follows a Beta distribution $\operatorname{Beta}(1.0,5.0)$ on $[0,0.1 \mu \mathrm{m}]$, which leads to a probability higher than $98 \%$ to have $d_{\min }<0.057 \mu \mathrm{m}$.

\subsection{Basic spatial information of fibers}

The cross section of UD-fiber reinforced matrix is studied based on the statistical analysis of spatial point patterns. We first explain the statistical characteristics that are considered and then illustrate them on the studied material system.

\subsubsection{The statistical characteristics of micro-structures for SVEs generator}

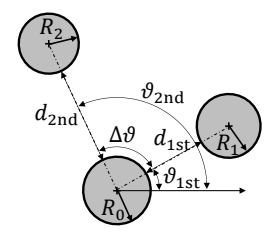

Figure 5: The first and second nearest-neighbor net distances and their orientations.

Some of the often used statistic functions are the point density, $\lambda$, the nearest-neighbor distance distribution function $D(r)$, the J-function, $J(r)$, the Ripleys K-function, $K(r)$, and so on [52]. In this work, the purpose of the spatial analysis is to be able to generate the SVEs, which have the same statistical

\footnotetext{
${ }^{1}$ In this work the roundness is evaluated from the perimeter $p$ and the cross-section area $A$ of the fibers following roundness $=\frac{p^{2}}{4 \pi A}$ and characterizes how close the cross-section is from a circle. Practically, the image analysis software Leica QWin ${ }^{\mathrm{TM}}$ was used to measure the perimeter and area parameters (the perimeter of the fibres taken as the total length of the boundary of fibres) from the SEM measurements.
} 
characteristics as the micro-structures of the real material. To this end, besides the distribution of fiber radius $p_{R}(r)$, we consider four other statistic functions related to the fibers spatial organization:

1. The nearest-neighbor net distance distribution function, which can be written as $p_{d_{1 \mathrm{st}}}(d)$, with $l=\left(d_{1 \mathrm{st}}+R_{0}+R_{1}\right)$, where $R_{0}$ and $R_{1}$ are the two neighboring fibers' radii, respectively and $l$ the distance between the fibers' centers; By using the net distance, the effect of fiber's radius on the nearest-neighbor distance can be excluded, which allows us to use independent variables to represent the fibers radii and their neighbor distances;

2. The distribution of the orientation of the un-directed line connecting the center points of a fiber and of its nearest neighbor, $p_{\vartheta_{1 \mathrm{st}}}(\theta)$ on $(-\pi, \pi]$; The distribution of orientation is a relevant issue for anisotropic point patterns, which might be the case in composite laminates; This orientation together with the net distance can have a critical effect on the mechanical response of volume elements;

3. The distribution $p_{\Delta d}(d)$ of the difference between the net distances to the second and to the first nearest-neighbors with $\Delta d=d_{2 \text { nd }}-d_{1 \text { st }}$; Since $\Delta d \geq 0$ by definition, using the distribution $p_{\Delta d}(d)$ ensures that the generated random variables satisfy $d_{2 \text { nd }} \geq d_{1 \text { st }}$;

4. The distribution $p_{\Delta \vartheta}(\theta)$ on $(0,2 \pi]$ of the difference $\Delta \vartheta=\vartheta_{2 \text { nd }}-\vartheta_{1 \text { st }}$ of the orientations $\vartheta_{2 \text { nd }}$ and $\vartheta_{1 \text { st }}$ of the second and the first nearest-neighbors; Therefore, the second nearest-neighbor is located referring to the first nearest-neighbor.

The first and second nearest-neighbor net distances and their orientations are illustrated in Fig. 5.

\subsubsection{The statistical analysis results based on SEM images}

The information of the first and second nearest-neighbor net distances and their orientations are extracted from the SEM images obtained with the amplification ratios of $2000 \times$ and $3000 \times$. The histograms of their distributions $p_{d_{1 \mathrm{st}}}(d), p_{\vartheta_{1 \mathrm{st}}}(\theta), p_{\Delta d}(d)$, and $p_{\Delta \vartheta}(\theta)$ are presented in Fig. 6. The correlation matrix of the four random variables, $d_{1 \mathrm{st}}, \vartheta_{1 \mathrm{st}}, \Delta d$, and $\Delta \vartheta$, reads

$$
\text { Cor }=\begin{array}{cccc}
d_{1 \mathrm{st}} & \vartheta_{1 \mathrm{st}} & \Delta d & \Delta \vartheta \\
d_{1 \mathrm{st}} \\
\vartheta_{1 \mathrm{st}} \\
\Delta d \\
\Delta \vartheta
\end{array}\left(\begin{array}{cccc}
1.0 & 0.014 & 0.205 & 0.022 \\
& 1.0 & 0.002 & 0.020 \\
\text { symmetric } & & 1.0 & -0.005 \\
& & & 1.0
\end{array}\right)
$$

where, each entry of Cor is the Pearson's correlation coefficient, i.e. Cor $(X, Y)=$ $\frac{\mathbb{E}[(X-\mathbb{E}(X))(Y-\mathbb{E}(Y))]}{\sigma_{X} \sigma_{Y}}$ the covariance of two variables divided by the product of their standard deviations $\sigma$., with $\mathbb{E}(\cdot)$ the expectation.

Figure 6 shows that the distributions of the four spatial parameters $d_{1 \text { st }}, \vartheta_{1 \text { st }}$, $\Delta d$, and $\Delta \vartheta$ all exhibit non-Gaussianity. Therefore, the statistical dependence 


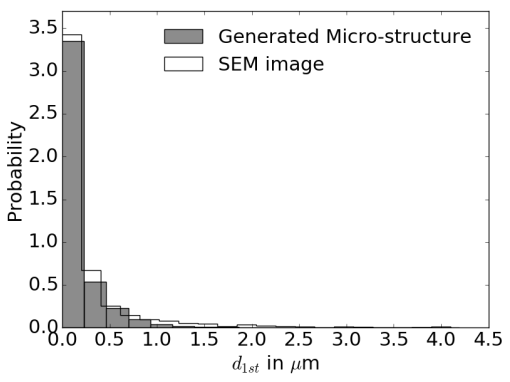

(a) Nearest-neighbor net distance

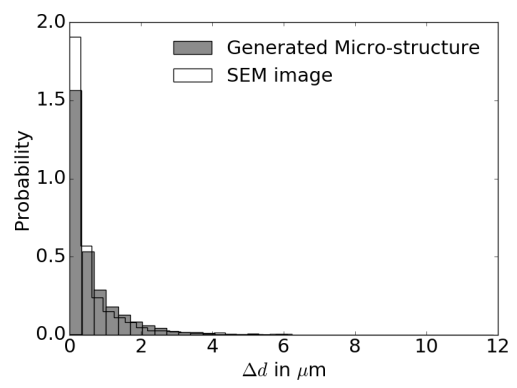

(c) Net distances difference

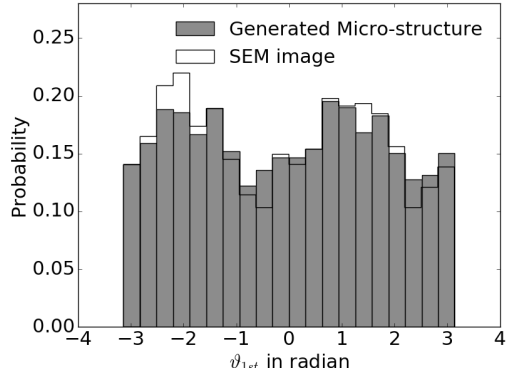

(b) Nearest-neighbor orientation

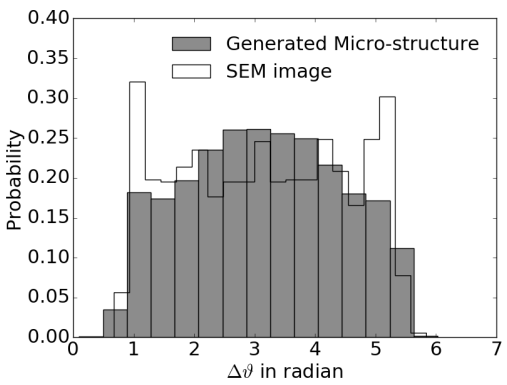

(d) Difference between nearest-neighbor orientations

Figure 6: The comparison of histograms of the four parameters characterizing the fiber spatial pattern obtained from the SEM images and the generated micro-structures (initial fibers/seeds number $N=4$ and maximum trying times $n_{\max }=5$ ): (a) Nearest-neighbor net distance $d_{1 \text { st }}$ distributions; (b) Nearest-neighbor orientation $\vartheta_{1 \text { st }}$ distribution; (c) Difference between the net distances to the second and to the first nearest-neighbors $\Delta d$ distribution; and (d) Difference between nearest-neighbor orientations $\Delta \vartheta$ distribution.

of these random variables can only be measured by their distance correlations matrix which reads for the four random variables

$$
\begin{aligned}
& \begin{array}{llll}
d_{1 \mathrm{st}} & \vartheta_{1 \mathrm{st}} \quad \Delta d \quad \Delta \vartheta
\end{array} \\
& \mathrm{dCor}=\begin{array}{c}
d_{1 \text { st }} \\
\vartheta_{1 \text { st }} \\
\Delta d \\
\Delta \vartheta
\end{array}\left(\begin{array}{cccc}
1.0 & 0.040 & 0.273 & 0.075 \\
& 1.0 & 0.048 & 0.046 \\
\text { symmetric } & & 1.0 & 0.064 \\
& & & 1.0
\end{array}\right) .
\end{aligned}
$$

Each entry of $\mathbf{d} \operatorname{Cor}(X, Y)$ is the distance covariance divided by the product of their distance standard deviations, whose detailed expressions can be found in reference [53].

The non-zero distance correlation coefficients of two statistical independent random variables may come from the limited number of samples. Hence, two random variables with a distance correlation coefficient smaller than 0.1 are treated independently. However, accordingly to the distance correlation matrix (2), the dependency between the net distance to the first nearest neighbor, 
$d_{1 s t}$, and the difference between the net distances to the second and first nearest neighbors, $\Delta d$, cannot be neglected; they need to be treated as statistical dependent random variables.

\section{Numerical micro-structures generation}

In order to be able to study SVEs of arbitrary number and size in the next section, numerical micro-structures are generated in this section. In this work, the micro-structure is characterized by the five random variables $R, d_{1 \mathrm{st}}$, $\vartheta_{1 \mathrm{st}}, \Delta d$ and $\Delta \vartheta$, which were described in Sections 2.3 and 2.4. The generator of the numerical micro-structures is thus based on the numerical sampling of these random variables combined with a fibers additive process. The statistical characteristics of the generated micro-structures is then compared to the one evaluated directly from the SEM images.

\subsection{Generation of the random variable $R, d_{1 s t}, \Delta d, \vartheta_{1 s t}$, and $\Delta \vartheta$}

Pseudo-random samples of an independent random variable $X$ can be generated easily from its cumulative distribution function $F_{X}(x)=\int_{-\infty}^{x} p_{X}(s) \mathrm{d} s=$ $\mathbb{P}\{X \leq x\}$. Using the pseudo-random samples of a random variable $U$, which has a uniform distribution on $[0,1]$, a simple generating process can be expressed as

$$
X=F_{X}^{-1}(U) .
$$

The independent random variables $R, \vartheta_{1 \text { st }}$, and $\Delta \vartheta$ are thus generated following the process (3). Because the cumulative distributions of these three random variables are arbitrary and extracted from SEM measurements, the empirical cumulative distributions $F_{R}(r), F_{\vartheta_{1 \mathrm{st}}}(\theta)$ and $F_{\Delta \vartheta}(\theta)$ are used.

The pseudo-random samples of the two dependent random variables $d_{1 \text { st }}$ and $\Delta d$ can be generated by using their copula [54]. Using the empirical distributions $F_{d_{1 \mathrm{st}}}(d)$ and $F_{\Delta d}(d)$ of the random variables $d_{1 \mathrm{st}}$ and $\Delta d$, considering the probability integral transform of each variable leads to

$$
\left(U_{1}, U_{2}\right)=\left(F_{d_{1 \mathrm{st}}}(d), F_{\Delta d}(d)\right),
$$

where the components of the random vector $\left(U_{1}, U_{2}\right)$ have a uniform marginal distribution on $[0,1]$. The copula of $\left(d_{1 \mathrm{st}}, \Delta d\right)$ is defined as the joint cumulative distribution function of $\left(U_{1}, U_{2}\right)$, which reads

$$
\boldsymbol{C}\left(u_{1}, u_{2}\right)=\mathbb{P}\left\{U_{1} \leq u_{1}, U_{2} \leq u_{2}\right\} .
$$

Pseudo-random samples of $\left(U_{1}, U_{2}\right)$ are generated from the copula distribution $\boldsymbol{C}\left(u_{1}, u_{2}\right)$ and the required samples are then constructed following

$$
\left(d_{1 \mathrm{st}}, \Delta d\right)=\left(F_{d_{1 \mathrm{st}}}^{-1}\left(U_{1}\right), F_{\Delta d}^{-1}\left(U_{2}\right)\right) .
$$

The data-driven methodology described here above avoids the need of assuming a distribution type. The implementation of the method follows closely

the copula method described in [54]. The main difficulty from an implementation point of view is the hash table search algorithm required in Eq. (6), which is however well documented. 


\subsection{Micro-structures generation}

Using numerical samples of $R, d_{1 \mathrm{st}}, \Delta d, \vartheta_{1 \mathrm{st}}$, and $\Delta \vartheta$, a fibers additive procedure is developed to generate numerical micro-structures of UD composites. The generation process is first described before evaluating the effect of its parameters on the statistical content of the generated micro-structures.

\subsubsection{Generation procedure}

Initialize fibers generation conditions in target window:

- Set the target fiber volume fraction $V_{\mathrm{I}}$ and its current value $v_{\mathrm{I}}=0$

- Set the maximum number of attempts $n_{\max }$

- Generate seed fibers for $N$ fiber clusters,

current number of fibers in each cluster is $n_{k}=1(k=0,1, \ldots, N-I)$, initialize cluster index $k=0$ and central fiber $i_{k}=0$ in cluster $k$

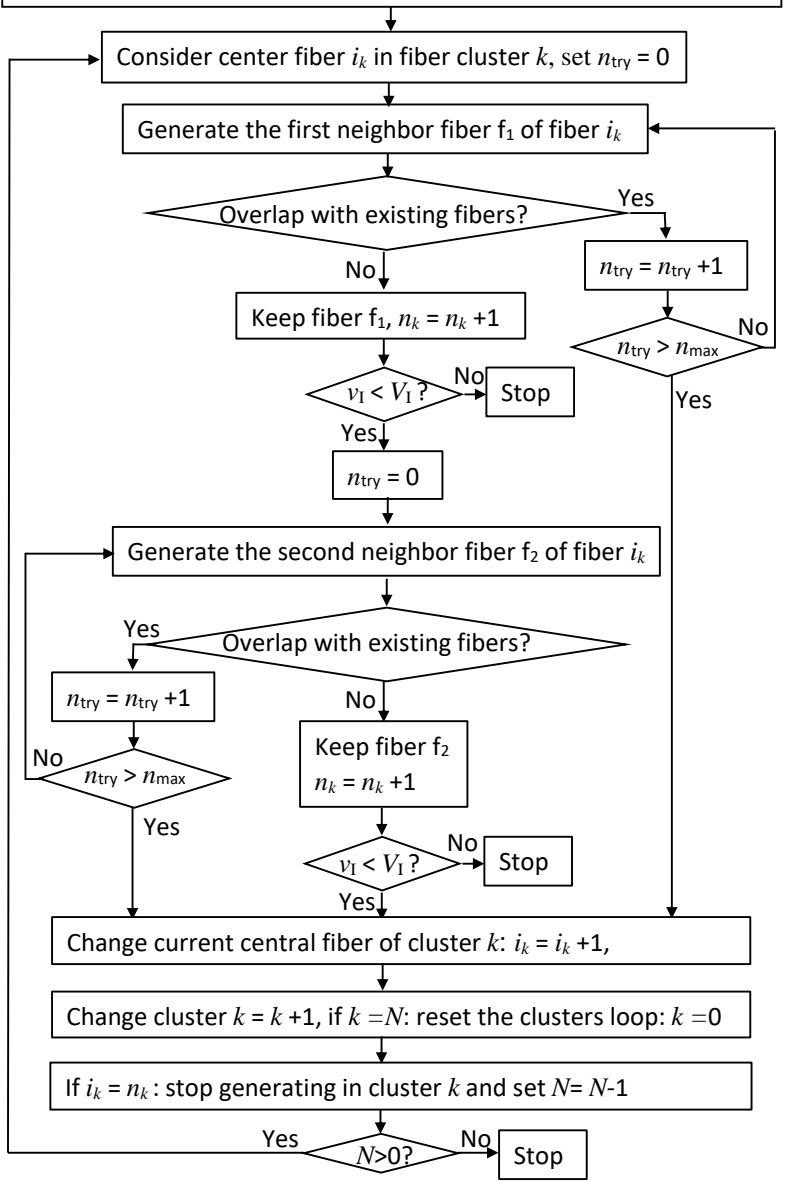

Figure 7: Flowchart of the micro-structure generation process. 


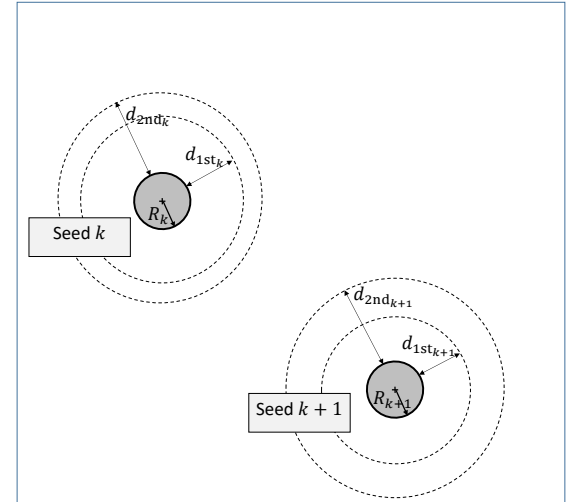

(a) Initialization

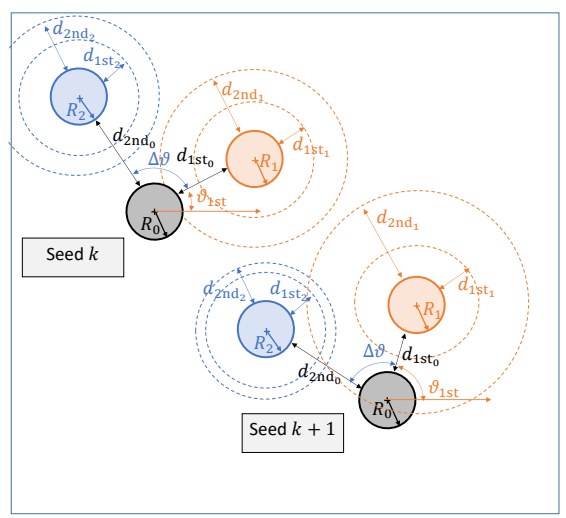

(c) After step $5,(k+1)^{\text {th }}$ loop iteration

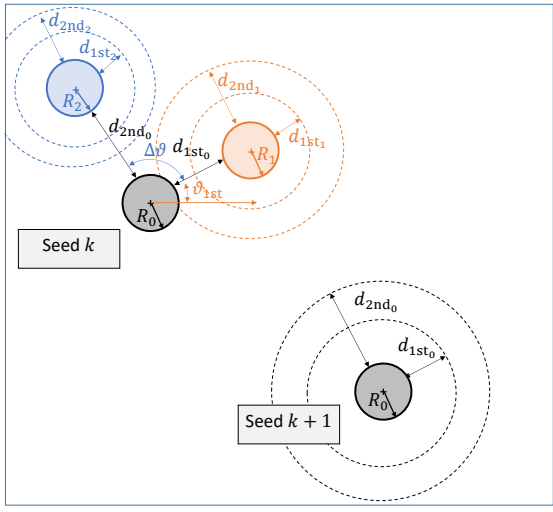

(b) After Step $5,(k)^{\text {th }}$ loop iteration

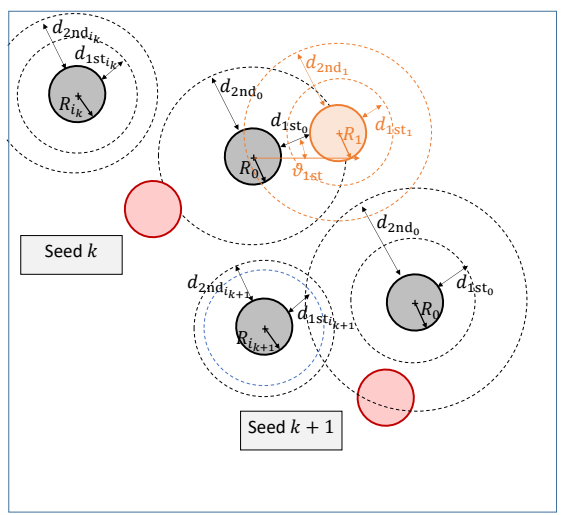

(d) After step 3, $(N+k)^{\text {th }}$ loop iteration

Figure 8: The micro-structure generation process in a square window. (a) After initialization, $N$ seed fibers are generated with their corresponding first and second nearest-neighbor net distances $d_{1 \text { st }}$ and $d_{2 \text { nd }}$; (b, c) After Step 5 of the $(k)^{\text {th }}(\mathrm{b})$ and $(k+1)^{\text {th }}$ (c) loop iterations, for each current central fiber in gray -here the seed fibers- the first and second nearest-neighbors, respectively in orange and blue, are generated from their respective orientations $\vartheta_{1 \mathrm{st}}$ and $\vartheta_{1 \text { st }}+\Delta \vartheta$ along with their corresponding first and second nearest-neighbor net distances $d_{1 \text { st }}$ and $d_{2 \text { nd }}$; (d) At step 6 , the previous central fibers (in red) are not considered anymore to generate new fibers and the newly generated fibers become the new current central fibers (in gray) used to generate the neighboring fibers, here the first one in orange after Step 3 of the $(N+1)^{\text {th }}$ loop iteration.

The fibers additive process is described by the following steps, see also the flowchart of Fig. 7.

1. Initialize fibers generation conditions:

- Set the size of the window and the target fiber volume fraction $V_{\mathrm{I}}^{2}$. Set the current fiber volume fraction $v_{\text {I }}$ to zero and define $n_{\max }$ the

${ }^{2}$ If the target fiber volume fraction $V_{\mathrm{I}}$ is set to 1 , the achieved volume fraction will depend 
maximum number of attempts.

- In the target window, generate $N$ initial fibers which are called the seed fibers. The realizations of random variables for one fiber include the radius $R_{i},(i=0,2, \ldots, N-1)$ of the fiber, its center $\left(x_{i}, y_{i}\right)$, $d_{1 \mathrm{st} i}$ and $\Delta d_{i}$, which gives $d_{2 \mathrm{nd} i}=d_{1 \mathrm{st} i}+\Delta d_{i}$. These seed fibers are illustrated in Fig. 8(a). Since each of these $N$ seed fibers will have their own first and second neighbors at the end of the generation process, the distance between any two fibers $i$ and $j$ needs to satisfy

$$
d_{i, j}=\sqrt{\left(x_{i}-x_{j}\right)^{2}+\left(y_{i}-y_{j}\right)^{2}} \geq R_{i}+R_{j}+\max \left(d_{2 \mathrm{nd} i}, d_{2 \mathrm{nd} j}\right),
$$

or in other words, their net distance cannot be smaller than the net distance to the second neighbor of any fiber.

- The generated fibers are divided into $N$ groups according to their corresponding seed fibers. The number of fibers in each group is initially $n_{k}=1$ and the initial central fiber $i_{k}=0$ of group $k$, from which the remaining fibers are generated, is the seed fiber.

2. Loop on the $N$ groups of fibers until the volume fraction of fibers reaches its target value $V_{\mathrm{I}}$ or until no more fiber can be added:

Step 1 For the fibers group $k, k \in[0, N-1]$, consider the central fiber $i_{k}$ which has its center located at $\left(x_{0}, y_{0}\right)$, a radius $R_{0}$, and the net distance functions $d_{1 \text { st } 0}, \Delta d_{0}$ and $d_{2 \text { nd } 0}$ (we omit the subscript $k$ for conciseness), see Fig. 8(b). Set the trying times counter $n_{\text {try }}=0$.

Step 2 Generate the first neighbor of the current present central fiber along with its four random variables, the fiber radius $R_{1}$, the orientation $\vartheta_{1 \text { st }}$ referring to the central fiber, and the net distances $d_{1 \text { st } 1}$ and $d_{2 \text { nd } 1}$ for its own two nearest neighbors that will be created later on. These random variables are generated from their empirical distributions and copula described in Section 3.1.

Step 3 Check the validity of the temporary fiber of radius $R_{1}$ centered at $\left(x_{1}, y_{1}\right)$, where $x_{1}=x_{0}+l \cos \left(\vartheta_{1 \mathrm{st}}\right), y_{1}=y_{0}+l \sin \left(\vartheta_{1 \mathrm{st}}\right)$ and $l=$ $R_{0}+R_{1}+d_{1 \text { st } 0}$.

a. If this fiber is at least partially inside the window, and if with respect to any other generated fiber $j$ at the exception of its corresponding central fiber it satisfies

$$
d_{1, j}=\sqrt{\left(x_{1}-x_{j}\right)^{2}+\left(y_{1}-y_{j}\right)^{2}} \geq R_{1}+R_{j}+\max \left(d_{2 \mathrm{nd} 1}, d_{2 \mathrm{nd} j}\right),
$$

then this fiber is accepted and $n_{k}=n_{k}+1$.

on the statistical description only. However a lower volume fraction can be enforced in the generation process through this parameter. 
* If the volume fraction $v_{\mathrm{I}}<V_{\mathrm{I}}$, set $n_{\text {try }}=0$ and goto the next step (Step 4).

* Else, stop.

b. Else the fiber is rejected and

* If $n_{\text {try }}<n_{\max }$, with $n_{\max }$ the maximum trying times, then $n_{\text {try }}=n_{\text {try }}+1$ and go back to Step 2 .

* If $n_{\text {try }}=n_{\max }$, then goto Step 6 .

Step 4 Generate the second neighbor of the current central fiber along with its four random variables, the fiber radius $R_{2}$, its orientation $\Delta \vartheta$, and the net distances $d_{1 \text { st } 2}$ and $d_{2 \text { nd } 2}$ for its own two nearest neighbors that will be created later on.

Step 5 Check the validity of the temporary fiber of radius $R_{2}$ centered at $\left(x_{2}, y_{2}\right)$, where $x_{2}=x_{0}+l \cos \left(\vartheta_{1 \mathrm{st}}+\Delta \vartheta\right), y_{2}=y_{0}+l \sin \left(\vartheta_{1 \mathrm{st}}+\Delta \vartheta\right)$ and $l=R_{0}+R_{2}+d_{1 \mathrm{st}}+\Delta d$.

a. If this fiber is at least partially inside the window, and if with respect to any other generated fiber $j$ at the exception of its corresponding central fiber it satisfies

$$
d_{2, j}=\sqrt{\left(x_{2}-x_{j}\right)^{2}+\left(y_{2}-y_{j}\right)^{2}} \geq R_{2}+R_{j}+\max \left(d_{2 \mathrm{nd} 2}, d_{2 \mathrm{nd} j}\right),
$$

then this fiber is accepted and $n_{k}=n_{k}+1$. An illustration of the fibers generated at the end of this step after the $(k)^{\text {th }}$ iteration of the loop can be seen in Fig. 8(b).

* If the volume fraction $v_{\mathrm{I}}<V_{\mathrm{I}}$, then goto Step 6 .

* Else, stop.

b. Else the fiber is rejected and

$*$ If $n_{\text {try }}<n_{\text {max }}$, then $n_{\text {try }}=n_{\text {try }}+1$ and go back to Step 4 .

$*$ If $n_{\text {try }}=n_{\text {max }}$, goto Step 6 .

Step 6 Change the current central fiber of the treated group $k$ with $i_{k}=i_{k}+1$ and move to the next group of fibers with $k=k+1$ (an illustration of the fibers generated at the end of Step 5 for $(k+1)^{\text {th }}$ iteration of the loop can be seen in Fig. 8(c)).

a. If $k=N$ then reset the loop on groups with $k=0$. Because the central fiber of a treated group $k$ was changed, the fibers will be added from a newly added fiber (an illustration of the fibers generated at the end of Step 3 for the $(N+k)^{\text {th }}$ iteration of the loop can be seen in Fig. 8(d)).

b. If $i_{k}=n_{k}$, all the fibers of group $k$ were considered as central fiber so stop adding fibers in group $k$ and decrease the total number of groups to loop on with $N=N-1$.

If $N=0$, no more fiber can be added, stop. Else, go back to Step 1.

Regarding to the additive procedure above, we draw the following remarks: 
1. Because of the assessment of the overlap criteria (8) and (9), the locations of the first and second nearest fibers are guaranteed to follow their statistical descriptions.

2. In the fibers additive procedure, only the location information of the two nearest neighboring fibers are used, therefore, the clustering phenomenon of fibers cannot be captured when considering only one seed. However, with $N>1$ initial/seed fibers, we have the possibility to represent this fibers clustering effect.

3. By increasing the maximum trying time $n_{\max }$, we increase the probability of obtaining higher volume fraction of fibers.

The effects of the two generator parameters $N$ and $n_{\max }$ are studied through generated micro-structure samples in the following section.

\subsubsection{The effects of the initial fibers number $N$ and of the maximum trying times $n_{\max }$.}

Since the values of $N$ and $n_{\max }$ mainly have effect on the fiber volume fraction obtained by the presented micro-structure generation procedure, this characteristic is studied in this section. The reference fiber volume fraction of $V_{\mathrm{I}}=0.502$ is an average value obtained from $103 \mathrm{SEM}$ images. Therefore, the generated average fiber volume fraction is computed from 104 numerical micro-structure realizations.

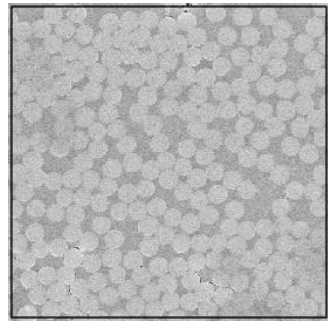

(a)

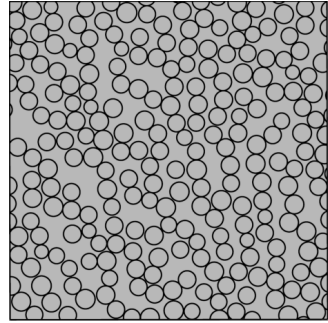

(b)

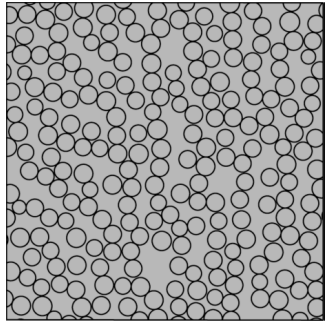

(c)

Figure 9: Cross section's micro-structures of UD-fiber reinforced composite: (a) one SEM image with an amplification ratio of $500 \times$; (b) and (c) two numerical micro-structure realizations $\left(N=4\right.$ and $\left.n_{\max }=5\right)$.

Firstly, 26 large windows of $200 \times 200 \mu \mathrm{m}^{2}$ were generated. To generate these large windows $\left(200 \times 200 \mu \mathrm{m}^{2}\right)$, different numbers of initial fibers $N=1,2,3$, and 4 are successively considered along with different numbers of maximum trying times, $n_{\max }=3,4,5,6$, and 7 . Figure 9 displays two large window numerical realizations obtained for the pair $\left(N, n_{\max }\right)=(4,5)$ along with an original SEM cross-section. Secondly, 4 non overlapping small windows were cut out from each large window. The size of these 104 small windows is $58 \times 37 \mu \mathrm{m}^{2}$, which is of comparable size to that of the SEM images with an amplification ratio of $3000 \times$. We then study the average fiber volume fractions obtained from the 104 small windows $\left(58 \times 37 \mu \mathrm{m}^{2}\right)$ for each pair of $\left(N, n_{\max }\right)$. This 
process is repeated three times for each pair $\left(N, n_{\max }\right)$ in order to ascertain the convergence of the statistical parameters.

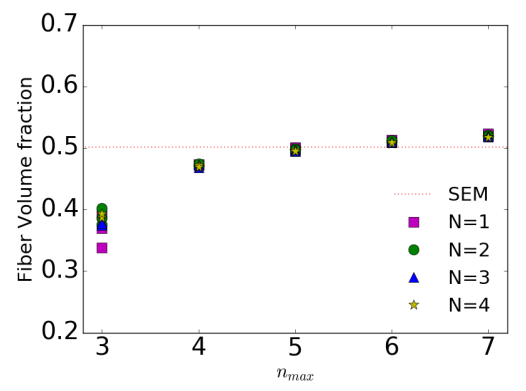

Figure 10: The average fiber volume fractions of generated micro-structures for different values of $N$ and $n_{\max }$.

Figure 10 shows that the three average fiber volume fractions reached for each pair $\left(N, n_{\max }\right)$ almost overlap each other for the higher values of $n_{\max }$. Moreover, the reached average volume fraction is governed by $n_{\max }$, and increases with the increase of $n_{\max }$. Although locally, such as in a small window, the fiber volume fraction can be found to be more than 0.6, the average fiber volume fraction has an upper limit which is about 0.52 in our case. This upper limit of the average volume fraction is defined by the spatial statistical characteristics of the fibers. As an example, with $N=4$ and $n_{\max }=5$, if we replace the generated neighboring fiber distances $d_{1 \text { st }}$ and $\Delta d$ by respectively $\alpha \times d_{1 \text { st }}$ and $\alpha \times \Delta d$ during the fibers additive process, the average fiber volume fraction can reach 0.54 with $\alpha=0.5$ and 0.59 with $\alpha=0.1$, respectively. However, if one wants to reach a higher fiber volume fraction by increasing the value of $n_{\max }$, this will not only require a long generation time, but also to change the spatial statistical characteristics of the fibers by rejecting the higher values of $d_{1 \text { st }}$ and $\Delta d$.

The effect of $N$ on the average fiber volume fraction is not obvious when $n_{\max } \geq 4$, since the results for different values of $N$ overlap each other. This is explained by the fact that the existence of fibers clustering corresponds to a low fiber volume fraction, while obvious gaps appear between the fibers clusters. If the phenomenon of fibers clustering is expected, the value of $N$ needs to be chosen depending on the size of the generated windows.

\subsection{The statistical characteristics of the generated micro-structures}

Since 103 SEM images were used to perform the spatial statistical analysis, the same spatial statistical analysis is also performed on the 104 generated micro-structures for each pair of $\left(N, n_{\max }\right)$.

The statistical characteristics of $R, d_{1 \mathrm{st}}, \vartheta_{1 \mathrm{st}}$, and $\Delta d$ is well recovered by the generated micro-structures for any values of $N$ and $n_{\max }(\leq 7)$. As an example, using the results for $N=4$ and $n_{\max }=5$, the histograms of $R, d_{1 \mathrm{st}}, \vartheta_{1 \mathrm{st}}$ and $\Delta d$, and of $\Delta \vartheta$ are plotted in Figs. 4, and 6, respectively, and compared to the 


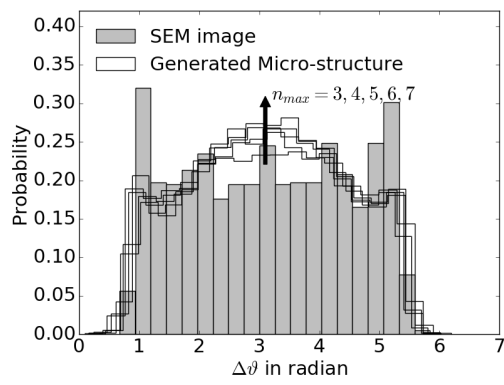

Figure 11: The histograms of $\Delta \vartheta$ of the generated micro-structures for $N=3$ and different values of $n_{\max }=3,4,5,6,7$.

histograms built from the SEM images. The only obvious difference can be seen for $\Delta \vartheta$ in Fig. $6(\mathrm{~d})$. Indeed, the generated $\Delta \vartheta$ for the second nearest neighbor can be rejected during the overlapping check and regenerated for several times $\left(\leq n_{\max }\right)$. Therefore, the resulting distribution of $\Delta \vartheta$ is affected by the value of $n_{\max }$. Figure 11 shows the histograms of $\Delta \vartheta$ obtained for different values of $n_{\max }=3,4,5,6,7$ and for $N=3$. The probability for $\Delta \vartheta$ to have a value around $\pi$ increases with the increase of $n_{\max }$. However, we assume that the differences between the histograms obtained from the SEM images and from the generated micro-structures are still acceptable for the further mechanical analyzes, since in the presented micro-structures generation procedure, to reach a reasonable fiber volume fraction, the constraint from this empirical distribution must be relaxed.

\subsection{Comparison of the second order characteristics Ripleys K-function}

The second-order Ripleys K-function, $K(r)$, is regarded as one of the most important tool for point pattern analysis [52]. Therefore we compare the Kfunctions evaluated from a SEM image and a generated micro-structure.

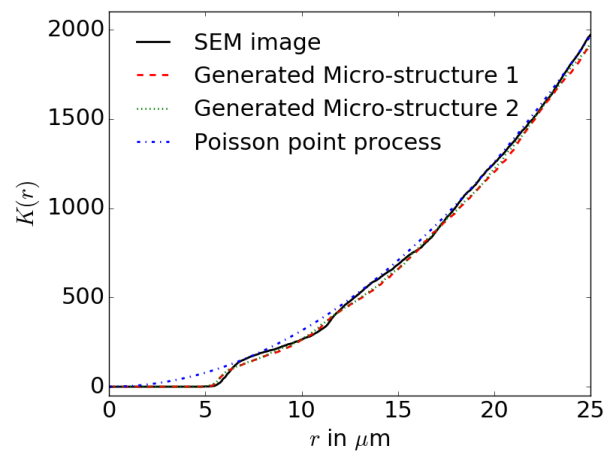

Figure 12: Comparison between the Ripleys K-functions evaluated from the SEM measurement and two numerical cross section realizations. 
Let $\lambda$ denotes the intensity or point density, which is the mean number of points per unit area. $\lambda K(r)$ is the average number of the other points found within the distance $r$ from the typical point. Let $n$ be the number of points in the observation window, and let $n_{i}(r),(i=1,2, \ldots, n)$, be the number of points within distance $r$ from the point $\boldsymbol{x}_{i}$, excluding $\boldsymbol{x}_{i}$ itself. Therefore, an estimator of $\lambda K(r)$ reads

$$
\lambda K(r)=\frac{1}{n} \sum_{i=1}^{n} n_{i}(r),
$$

and for the Poisson process, the K-function has a simple form,

$$
K(r)=\pi r^{2} .
$$

Considering the SEM image and the two micro-structure realizations illustrated in Fig. 9, a good agreement of their K-functions can be seen in Fig. 12, in which the K-function of Poisson point process is also presented as a reference.

In Fig. 9, it can be seen that the main features of the real micro-structure (Fig. 9(a)) are well represented by the generated micro-structures (Figs. 9(b) and $9(\mathrm{c}))$. However, in the generated micro-structures, the fiber distribution is more regular than in the SEM image. This difference comes from the assumption of stationary or homogeneous point process. The spatial statistical information, the distributions of the random variables $d_{1 \mathrm{st}}, \vartheta_{1 \mathrm{st}}, \Delta d$, and $\Delta \vartheta$, was obtained from the analysis of 103 SEM images, in the sense of average. In fact, the point patterns are inhomogeneous for small observation windows and become more homogeneous with the increase of the windows size. This trend can be seen in Fig. 12, in which with the increase of $r$, the function $K(r)$ of the micro-structures become closer to that of the Poisson point process. The inhomogeneous point process can also be generated under the presented generating procedure. However, more detailed spatial statistical descriptions are required, such as random field descriptions for $d_{1 \text { st }}, \vartheta_{1 \text { st }}, \Delta d$ and $\Delta \vartheta$ instead of considering them as random variables. A simple example of inhomogeneous point process is given in Appendix A.

In addition, the real micro-structure of UD fiber reinforced composites' crosssection does not follow a stationary spatial point pattern when the observation scale increases [55]. There always exists resin rich area, especially at the interface of two laminates. In order to have a complete statistical spatial description of the real micro-structures, not only the distributions of the fibers are required, but also the information related to micro-defects and resin rich areas. This means that a large amount of digital images at different length scales are needed.

\section{Stochastic study of composite materials from the generated SVEs}

In this section, the elastic properties of UD fiber reinforced composites are studied in a probabilistic way. First, the computational homogenization theory is summarized. Stochastic homogenization is then applied on generated SVEs of reduced dimensions. In particular, the effects of the SVEs size and 
of the boundary conditions applied during the homogenization process on the homogenized elastic behavior are studied. One advantage of the micro-structure generator is the ability to generate an arbitrary number of SVEs of arbitrary sizes having a non-constant volume fraction of fibers. Finally, with a view to the use of macro-scale stochastic finite elements, different stochastic interpolations methods are studied in order to represent the probabilistic behavior of large SVEs of dimensions corresponding to the size of macro-scale finite elements.

\subsection{Evaluation of the apparent elastic properties of the meso-scale homogenized material}

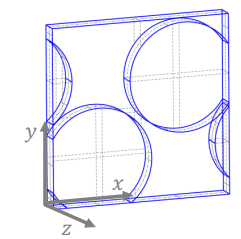

(a)

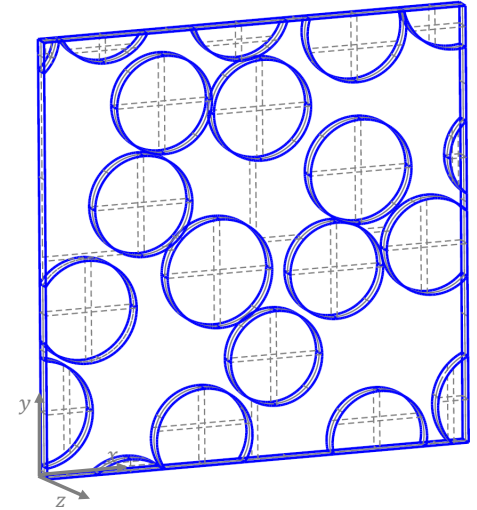

(c)

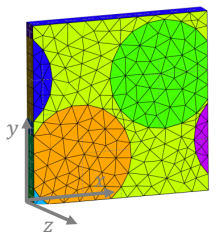

(b)

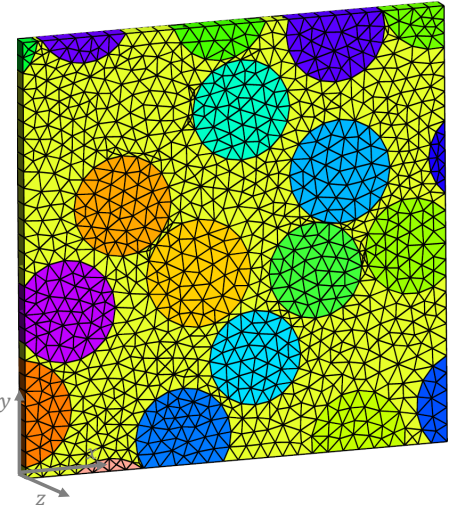

(d)

Figure 13: Examples of meso-scale volume elements $\omega$ : (a) geometry of a $10 \times 10 \times 1 \mu \mathrm{m}^{3} \mathrm{SVE}$ realization; (b) finite element discretization of the $10 \times 10 \times 1 \mu \mathrm{m}^{3} \mathrm{SVE}$; (c) geometry of a $25 \times 25 \times 1 \mu \mathrm{m}^{3} \mathrm{SVE}$ realization; and (d) finite element discretization of the $25 \times 25 \times 1 \mu \mathrm{m}^{3}$ SVE.

The apparent -or homogenized- meso-scale material tensor is estimated from the finite element resolution of the meso-scale boundary value problem (BVP) $[23,56]$. In the following, we summarize the scale transition equations in the context of linear elasticity. In particular, we define the different kinds of boundary conditions that can be applied on the meso-scale volume element with a particular emphasis on the extraction of the homogenized material operators following the multiple-constraint projection method [57] detailed in [58]. 


\subsubsection{The micro-scale problem}

The micro-scale problem is defined on the meso-scale volume element, here an SVE, $\omega$ of boundary $\partial \omega$, see Fig. 1. The SVE is built as a 3D model extruded as a parallelepiped from the cross-section generated in Section 3. The $z$-direction refers to the longitudinal direction " $\mathrm{L}$ " of the fibers, and the $x$ - and $y$-directions refer to the transverse directions " $\mathrm{T}$ " and " $\mathrm{T}$ " " of the fibers with $y$ along the thickness direction of the laminate, see Fig. 13.

If the SVE is small enough for the time of the strain wave to propagate in the SVE to remain negligible, the equivalence of the micro-strain to the macro-strain is instantaneous and the equilibrium equations read

$$
\left\{\begin{array}{l}
\nabla_{\mathrm{m}} \cdot \boldsymbol{\sigma}_{\mathrm{m}}=\mathbf{0} \quad \forall \boldsymbol{x} \in \omega, \\
\boldsymbol{n}_{\mathrm{m}} \cdot \boldsymbol{\sigma}_{\mathrm{m}}=\boldsymbol{t}_{\mathrm{m}} \quad \forall \boldsymbol{x} \in \partial \omega,
\end{array}\right.
$$

where the subscript ' $m$ ' refers to the local value at the micro-scale, $\sigma_{\mathrm{m}}$ is the Cauchy stress tensor, and $\boldsymbol{t}_{\mathrm{m}}$ is the surface traction on the boundary of outward unit normal $\boldsymbol{n}_{\mathrm{m}}$.

The micro-scale problem is completed by the local constitutive laws of the different materials. In this work we assume (possibly anisotropic) linear elasticity leading to

$$
\sigma_{\mathrm{m}}=\mathbb{C}_{\mathrm{m}}(\boldsymbol{x}): \varepsilon_{\mathrm{m}},
$$

where $\mathbb{C}_{\mathrm{m}}(\boldsymbol{x})$ is a fourth-order material tensor, which depends on the micro-scale material point location $\boldsymbol{x}$, and $\varepsilon_{\mathrm{m}}=\frac{1}{2}\left(\nabla_{\mathrm{m}} \otimes \boldsymbol{u}_{\mathrm{m}}+\boldsymbol{u}_{\mathrm{m}} \otimes \nabla_{\mathrm{m}}\right)$ is the smalldeformation strain tensor evaluated in terms of the micro-scale displacement $\boldsymbol{u}_{\mathrm{m}}$.

\subsubsection{The scale transition}

Homogenized values are defined as the volume average of a micro-scale field on the meso-scale volume-element $\omega$ by

$$
\cdot \mathrm{M}=<\cdot \mathrm{m}>=\frac{1}{V(\omega)} \int_{\omega} \cdot{ }_{\mathrm{m}} \mathrm{d} V
$$

where the subscript ' $\mathrm{M}$ ' refers to the homogenized value, $\langle\cdot\rangle$ is the volume average of the field $\cdot$, and $V(\omega)$ is the volume of the meso-scale volume element $\omega$. In particular, in the context of linear elasticity, the homogenized stress tensor $\sigma_{\mathrm{M}}$ and strain tensor $\varepsilon_{\mathrm{M}}$ respectively read

$$
\left\{\begin{array}{l}
\sigma_{\mathrm{M}}=<\boldsymbol{\sigma}_{\mathrm{m}}>=<\mathbb{C}_{\mathrm{m}}: \varepsilon_{\mathrm{m}}>=\mathbb{C}_{\mathrm{M}}: \varepsilon_{\mathrm{M}}, \\
\varepsilon_{\mathrm{M}}=\left(\frac{\nabla_{\mathrm{M}} \otimes \boldsymbol{u}_{\mathrm{M}}+\boldsymbol{u}_{\mathrm{M}} \otimes \nabla_{\mathrm{M}}}{2}\right)=<\varepsilon_{\mathrm{m}}>,
\end{array}\right.
$$

where $\mathbb{C}_{\mathrm{M}}$ is the apparent fourth-order material tensor and $\boldsymbol{u}_{\mathrm{M}}$ is the macroscale displacement field.

In general $\mathbb{C}_{\mathrm{M}}$, the apparent fourth-order material tensor, is not the volume average of $\mathbb{C}_{\mathrm{m}}$. Instead, it should be defined in order to ensure the energy consistency at the different scales, which corresponds to the Hill-Mandel condition

$$
\sigma_{\mathrm{M}}: \delta \varepsilon_{\mathrm{M}}=\delta \varepsilon_{\mathrm{M}}: \mathbb{C}_{\mathrm{M}}: \varepsilon_{\mathrm{M}}=<\delta \varepsilon_{\mathrm{m}}: \mathbb{C}_{\mathrm{m}}: \varepsilon_{\mathrm{m}}>
$$


The scale transition problem is completed by the definition of the boundary conditions applied on the meso-scale volume element $\omega$. To this end, the microscale displacement field is written under the form

$$
\boldsymbol{u}_{\mathrm{m}}(\boldsymbol{x})=\left(\boldsymbol{u}_{\mathrm{M}} \otimes \nabla_{\mathrm{M}}\right) \cdot\left(\boldsymbol{x}-\boldsymbol{x}_{\mathrm{ref}}\right)+\boldsymbol{u}^{\prime}(\boldsymbol{x}),
$$

where $\boldsymbol{u}^{\prime}$ is the perturbation field and $\boldsymbol{x}_{\text {ref }}$ a reference point of $\omega$. In order to satisfy Eq. (15) this perturbation field should satisfy

$$
0=<\boldsymbol{u}^{\prime}(\boldsymbol{x}) \otimes \nabla_{\mathrm{m}}>=\frac{1}{V(\omega)} \int_{\partial \omega} \boldsymbol{u}^{\prime} \otimes \boldsymbol{n}_{\mathrm{m}} \mathrm{d} S .
$$

Moreover, the Hill-Mandel condition can be rewritten by substituting Eq. (17) in Eq. (16), integrating by parts, and using the equilibrium Eqs. (12), which lead to

$$
\boldsymbol{\sigma}_{\mathrm{M}}: \delta \varepsilon_{\mathrm{M}}=<\boldsymbol{\sigma}_{\mathrm{m}}: \delta \varepsilon_{\mathrm{m}}>=\boldsymbol{\sigma}_{\mathrm{M}}: \delta \varepsilon_{\mathrm{M}}+\frac{1}{V(\omega)} \int_{\partial \omega}\left(\boldsymbol{\sigma}_{\mathrm{m}} \cdot \boldsymbol{n}\right) \cdot \delta \boldsymbol{u}^{\prime} \mathrm{d} S,
$$

or again to

$$
0=\int_{\partial \omega} \boldsymbol{t}_{\mathrm{m}} \cdot \delta \boldsymbol{u}^{\prime} \mathrm{d} S
$$

\subsubsection{Definition of the constrained micro-scale finite element problem}

The weak form associated to the micro-scale equations (12) reads

$$
\int_{\omega} \boldsymbol{\sigma}_{\mathrm{m}}:\left(\delta \boldsymbol{u}^{\prime} \otimes \nabla_{\mathrm{m}}\right) \mathrm{d} V=0, \quad \forall \delta \boldsymbol{u}^{\prime} \in \mathcal{U},
$$

where $\mathcal{U}$ is the admissible kinematic field defined as a subset of the kinematic field satisfying (18). In that case the Hill-Mandel condition (20) is always verified [58-60]. Therefore, the admissible kinematic field $\mathcal{U}$ is defined by specific boundary conditions whose constraint is to satisfy (18). In this work we consider the following kinds

1. The Periodic Boundary Conditions (PBCs) read

$$
\left\{\begin{array}{c}
\boldsymbol{u}_{\mathrm{m}}\left(\boldsymbol{x}^{+}\right)-\boldsymbol{u}_{\mathrm{m}}\left(\boldsymbol{x}^{-}\right)=\left(\boldsymbol{u}_{\mathrm{M}} \otimes \nabla_{\mathrm{M}}\right) \cdot\left(\boldsymbol{x}^{+}-\boldsymbol{x}^{-}\right), \\
\forall \boldsymbol{x}^{+} \in \partial \omega^{+} \text {and corresponding } \boldsymbol{x}^{-} \in \partial \omega^{-},
\end{array}\right.
$$

where the parallelepiped SVE faces have been separated in opposite surfaces $\partial \omega^{-}$and $\partial \omega^{+}$. Equation (22) satisfies (18).

2. The Static Uniform Boundary Conditions (SUBCs) are formally written

$$
\boldsymbol{t}_{m}=\boldsymbol{n}_{\mathrm{m}} \cdot \boldsymbol{\sigma}_{\mathrm{M}} \quad \forall \boldsymbol{x} \in \partial \omega,
$$

and allow rewriting the Hill-Mandel condition (20) as

$$
0=\int_{\partial \omega} \boldsymbol{t}_{\mathrm{m}} \cdot \boldsymbol{u}_{\mathrm{m}}^{\prime} \mathrm{d} S=\boldsymbol{\sigma}_{\mathrm{M}}: \int_{\partial \omega} \boldsymbol{n}_{\mathrm{m}} \otimes \boldsymbol{u}^{\prime} \mathrm{d} S .
$$


This condition is satisfied by considering the Zero Average Fluctuation Boundary Conditions (ZAFBCs) on each face $S$ of the meso-scale volume element

$$
\int_{S} \boldsymbol{u}^{\prime} \mathrm{d} S=0
$$

which also satisfy Eq. (18) for meso-scale volume elements having flat faces. In this work we consider the constraint (25) and use the SUBC denomination by abuse of language.

3. The Kinematic Uniform Boundary Conditions (KUBCs) for which there is no fluctuation on the boundary, i.e.

$$
\boldsymbol{u}^{\prime}=0 \quad \forall \boldsymbol{x} \in \partial \omega,
$$

which directly satisfy (18). However, when considering a 3D meso-scale volume element of UD composite material, in which the fibers are oriented along the $z$-direction, applying KUBCs on the two opposite faces $S_{\mathrm{L}}^{+}$and $S_{\mathrm{L}}^{-}$perpendicular to the fibers results in an overstiff Voigt assumption. Therefore we apply the following conditions

$$
\left\{\begin{array}{l}
\boldsymbol{u}^{\prime}=0 \quad \forall \boldsymbol{x} \in S_{\mathrm{T}}^{+}, S_{\mathrm{T}}^{-}, S_{\mathrm{T}}^{+}, S_{\mathrm{T}}^{-}, \\
u_{z}^{\prime}=0 \quad \forall \boldsymbol{x} \in S_{\mathrm{L}}^{+}, S_{\mathrm{L}}^{-}, \\
\int_{S_{\mathrm{L}}^{+}} u_{x}^{\prime} \mathrm{d} S=\int_{S_{\mathrm{L}}^{-}} u_{x}^{\prime} \mathrm{d} S=\int_{S_{\mathrm{L}}^{+}} u_{y}^{\prime} \mathrm{d} S=\int_{S_{\mathrm{L}}^{-}} u_{y}^{\prime} \mathrm{d} S=0 .
\end{array}\right.
$$

\subsubsection{Resolution of the constrained micro-scale finite element problem}

The numerical resolution of the meso-scale BVP relies on the discretization of the meso-scale volume element $\omega$ in finite elements $\omega^{e}$, see Figs. 13(b) and 13(d). The finite element discretizations of (21) and of the constraints (22), (25) or (27) respectively lead to the set of coupled equations

$$
\left\{\begin{array}{l}
\mathbf{K}_{\mathrm{m}} \mathbf{u}_{\mathrm{m}}-\mathbf{C}^{T} \boldsymbol{\lambda}=0, \text { and } \\
\mathbf{C} \mathbf{u}_{\mathrm{m}}-\mathbf{S} \mathcal{E}_{\mathrm{M}}=0
\end{array}\right.
$$

where $\mathbf{K}_{\mathrm{m}}$ is the stiffness matrix of the unconstrained meso-scale BVP, $\mathbf{u}_{\mathrm{m}}$ is the vector of the nodal displacement, $\boldsymbol{\lambda}$ is the vector of the Lagrange multipliers, which enforce the constraints, $\mathcal{E}_{\mathrm{M}}$ represents the macro-scale kinematic variable $\varepsilon_{\mathrm{M}}$ written under a vector form, and where $\mathbf{C}$ and $\mathbf{S}$ are the so-called constraints matrix and kinematic matrix, respectively, built from the constraints (22), (25) or (27). In particular, in order to apply the PBCs on non-periodic microstructures, and thus on a non-periodic mesh, we have recourse to a polynomial interpolation of the unknowns fields on the boundary, as detailed in [61]. The stiffness matrix of the meso-scale finite element discretization (28) reads

$$
\mathbf{K}_{\mathrm{m}}=\bigwedge_{\omega^{e}} \int_{\omega^{e}}\left(\mathbf{B}^{e}\right)^{T} \mathcal{C}_{\mathrm{m}} \mathbf{B}^{e} \mathrm{~d} V
$$


where $\mathcal{C}_{\mathrm{m}}$ is the matrix notation of the fourth-order tensor $\mathbb{C}_{\mathrm{m}}, \mathbf{B}^{e}$ is the elementary matrix of the shape functions gradient, and where $\bigwedge_{\omega^{e}}$ is used to symbolize the assembly process.

Following the Lagrange multiplier elimination approach summarized in Appendix $\mathrm{B}$, the apparent elasticity tensor in the matrix form $\mathcal{C}_{\mathrm{M}}$ is obtained as

$$
\mathcal{C}_{\mathrm{M}}=\mathbf{D} \tilde{\mathbf{K}}_{\mathrm{m}}^{-1}\left(\mathbf{C}^{T}-\mathbf{Q}^{T} \mathbf{K}_{\mathrm{m}} \mathbf{C}^{T}\left(\mathbf{C C}^{T}\right)^{-1}\right) \mathbf{S} .
$$

In this equation $\mathbf{Q}=\mathbf{I}-\mathbf{C}^{T}\left(\mathbf{C C}^{T}\right)^{-1} \mathbf{C}, \tilde{\mathbf{K}}_{\mathrm{m}}=\mathbf{C}^{T} \mathbf{C}+\mathbf{Q}^{T} \mathbf{K}_{\mathrm{m}} \mathbf{Q}$, and $\mathbf{D}=$ $\left(\frac{1}{V(\omega)} \bigwedge_{\omega^{e}} \int_{\omega^{e}} \mathcal{C}_{\mathrm{m}} \mathbf{B}^{e} \mathrm{~d} V\right)$, see details in [58].

The homogenized material property can be treated as orthotropic, and the nine material parameters $E_{x}, E_{y}, E_{z}$-the three Young's moduli, $\nu_{x y}, \nu_{x z}, \nu_{y z}-$ the three Poisson coefficients, and $\mu_{x y}, \mu_{x z}, \mu_{y z}$-the three shear moduli- can be extracted from $\mathbb{C}_{\mathrm{M}}$ of each SVE, where the $z$ direction refers to the longitudinal direction "L" of fibers, and where $x$ and $y$ directions refer to the transverse directions " $\mathrm{T}$ " and " $\mathrm{T}$ " " of fibers with $y$ along the thickness direction of the laminate.

\subsection{Apparent elastic properties of Small SVEs (SSVEs)}

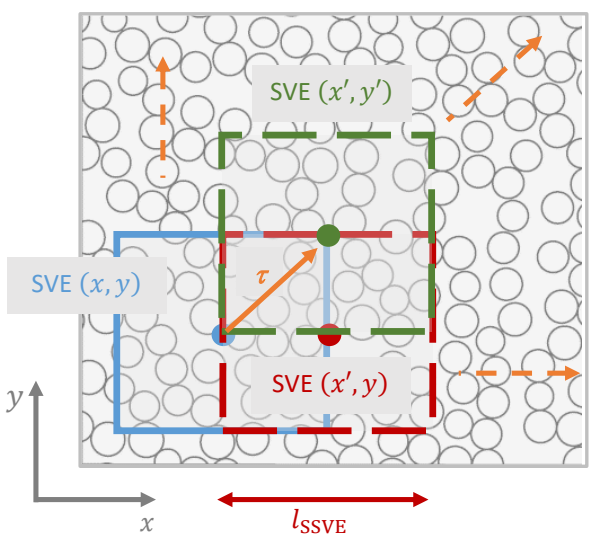

Figure 14: Extraction of SSVEs from a large micro-structure window; the two SSVE centers are separated by a vector $\tau$.

In order to study the effect of the SVE size and of the boundary conditions, in this section we consider parallelepiped SVEs of two different lengths: $l_{\mathrm{SSVE}}=$ $10 \mu \mathrm{m}$ and $l_{\mathrm{SSVE}}=25 \mu \mathrm{m}$, see examples of realizations in Fig. 13. The SVEs of reduced length are denoted as Small SVEs (SSVEs). All the SSVEs are cut from a larger micro-structure Big SVEs (BSVE) with an overlap with their neighbors by half of their length, see Figure 14, in order to capture the correlation effect.

The applied elastic properties of matrix and fibers have been identified in [48] for a similar material system and are: 
- Matrix: Elastic Young's modulus $E_{0}^{\mathrm{el}}=3.2 \mathrm{GPa}$; Poisson ratio $\nu_{0}=0.3$.

- Inclusions (fibers): Longitudinal Young's modulus $E_{\mathrm{L}}^{\mathrm{el}}=230 \mathrm{GPa}$; transverse Young's modulus $E_{\mathrm{TI}}^{\mathrm{el}}=40 \mathrm{GPa}$; transverse Poisson ratio $\nu_{\mathrm{TTI}}=$ 0.2 ; longitudinal-transverse Poisson ratio $\nu_{\mathrm{LTI}}=0.256$; transverse shear modulus $G_{\mathrm{TTI}}=16.7 \mathrm{GPa}$; longitudinal-transverse shear modulus $G_{\mathrm{LTI}}=$ $24 \mathrm{GPa}$.

\subsubsection{Statistic and stochastic characteristics}

On the one hand, one hundred BSVEs of size $50 \times 50 \times 1 \mu \mathrm{m}^{3}$ are generated and from each of them 81 overlapping SSVEs of length $l_{\mathrm{SSVE}}=10 \mu \mathrm{m}$ are extracted, which leads to totally 8100 SSVEs. On the other hand, one hundred BSVEs of size $100 \times 100 \times 1 \mu \mathrm{m}^{3}$ are generated and from each of them 49 overlapping SSVEs of length $l_{\mathrm{SSVE}}=25 \mu \mathrm{m}$ are extracted, which leads to totally 4900 SSVEs. In the following, since the depth of the SSVEs is always $1 \mu \mathrm{m}$, the information is no longer repeated and the apparent elastic properties of the SSVEs are evaluated with the computational homogenization scheme described in Section 4.1.

In this section we study the correlation information between neighboring SSVEs and we thus apply only PBCs on the SSVEs. The spatial cross-correlation $\boldsymbol{R}_{r s}(\boldsymbol{\tau})$ between two properties $r$ and $s$ of two SSVEs, whose centers are separated by a vector $\boldsymbol{\tau}$, is extracted using the window technique illustrated in Fig. 14 assuming the random fields of the apparent elasticity tensor field $\mathcal{C}_{\mathrm{M}}(\boldsymbol{x})$ and of the volume fraction $V_{f}$ are stationary, with

$$
R_{r s}(\boldsymbol{\tau})=\frac{\mathbb{E}[(r(\boldsymbol{x})-\mathbb{E}[r])(s(\boldsymbol{x}+\boldsymbol{\tau})-\mathbb{E}[s])]}{\sigma_{r} \sigma_{s}} .
$$

The associated correlation length of the stationary random field is defined by [62]

$$
l_{r s}=\frac{\int_{-\infty}^{\infty} R_{r s}(\tau) \mathrm{d} \tau}{R_{r s}(0)} .
$$

Note that in Eq. (31) we have assumed that the apparent properties are stationary since the generated micro-structures are such. We have however applied periodic boundary conditions on a non-periodic structure. Nevertheless, since we evaluate Eq. (31) considering several (one hundred) initial BSVEs from which the windows are extracted, we recover a stationary apparent property random field as well. More studies and discussions on the effect of different boundary conditions will be presented in the next section.

The histograms of the three in-plane apparent elastic properties of the SSVEs, $E_{x}, v_{x y}, \mu_{x y}$ and of the fiber volume fraction $V_{f}$ are presented in Figs. 15 and 16 , respectively. The fitted normal distributions of the histograms are reported, with the corresponding mean and variance and with the Kolmogorov-Smirnov goodness-of-fit of the normal distribution. From Figs. 15 and 16 we can see that, with the increase of the SSVE size, not only the variances of the elastic 

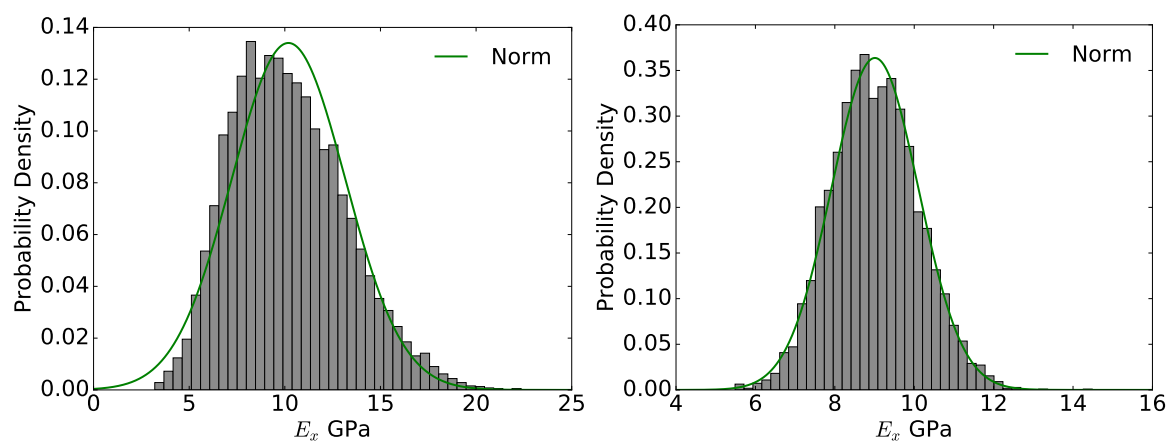

(a) $\mathbb{E}=10.19 \mathrm{GPa}, \sigma=2.98 \mathrm{Gpa}, \mathrm{K}-\mathrm{S}: 0.040$

(b) $\mathbb{E}=9.01 \mathrm{GPa}, \sigma=1.10 \mathrm{Gpa}, \mathrm{K}-\mathrm{S}: 0.017$
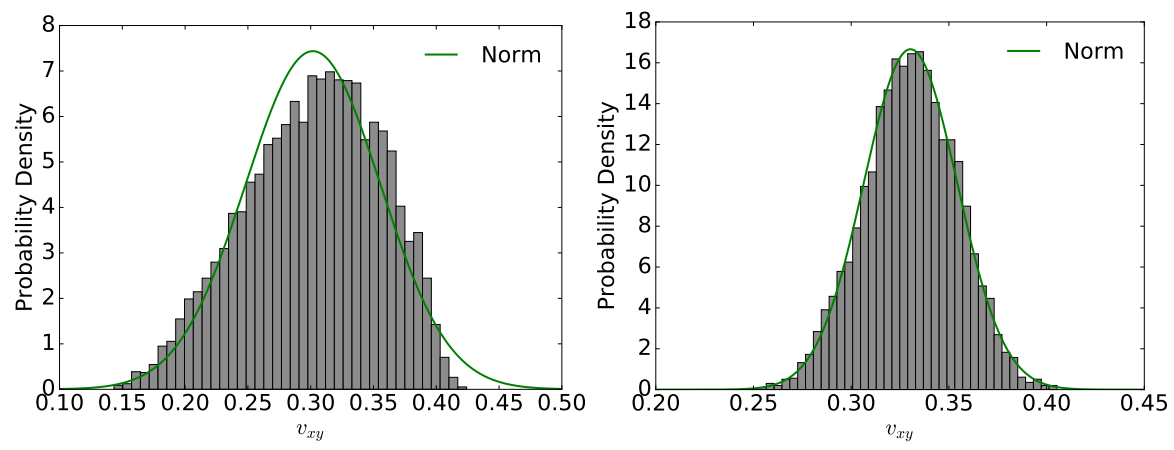

(c) $\mathbb{E}=0.302, \sigma=0.540, \mathrm{~K}-\mathrm{S}: 0.036$

(d) $\mathbb{E}=0.330, \sigma=0.024, \mathrm{~K}-\mathrm{S}: 0.012$
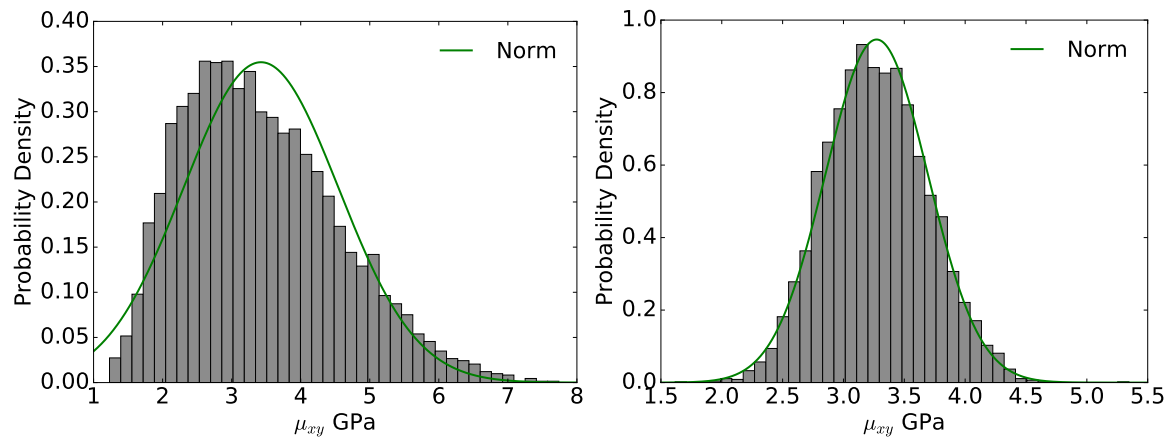

(e) $\mathbb{E}=3.42 \mathrm{GPa}, \sigma=1.12 \mathrm{Gpa}, \mathrm{K}-\mathrm{S}: 0.057$

(f) $\mathbb{E}=3.27 \mathrm{GPa}, \sigma=0.42 \mathrm{Gpa}, \mathrm{K}-\mathrm{S}: 0.017$

Figure 15: The histograms of the extracted SSVEs' apparent elastic properties: the left column (a), (c) and (e) refer to $l_{\mathrm{SSVE}}=10 \mu \mathrm{m}$ and the right column (b), (d) and (f) to $l_{\mathrm{SSVE}}=25 \mu \mathrm{m}$; fitted normal distributions are plotted for indication purpose with the mean value $\mathbb{E}$, standard deviation $\sigma$, and with the resulting Kolmogorov-Smirnov goodness-of-fit K-S.

properties and of the volume fraction distributions decrease, but also their distributions are getting closer to normal distributions as shown by the evolution of the goodness-of-fit. It bears emphasis that in this study we do not assume a 


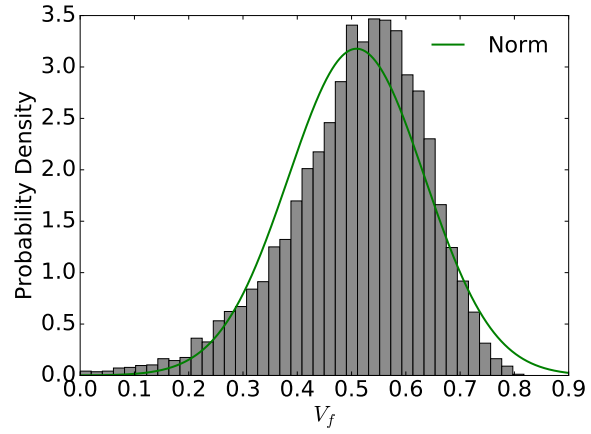

(a) $\mathbb{E}=0.509, \sigma=0.126, \mathrm{~K}-\mathrm{S}: 0.054$

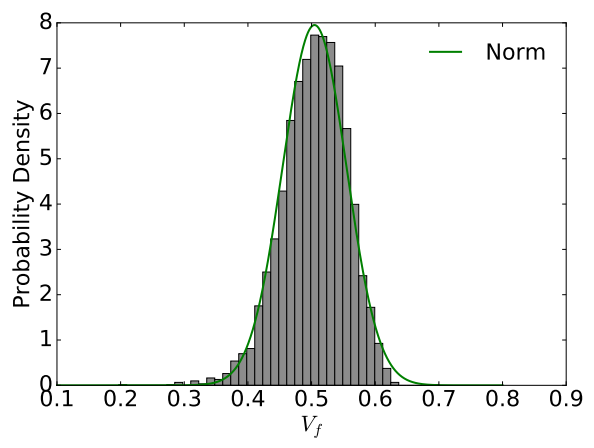

(b) $\mathbb{E}=0.504, \sigma=0.050, \mathrm{~K}-\mathrm{S}: 0.027$

Figure 16: The histograms of the extracted SSVEs' fiber volume fraction: (a) refers to $l_{\mathrm{SSVE}}=$ $10 \mu \mathrm{m}$ and (b) to $l_{\mathrm{SSVE}}=25 \mu \mathrm{m}$; fitted normal distributions are plotted for indication purpose with the mean value $\mathbb{E}$, standard deviation $\sigma$, and with the resulting Kolmogorov-Smirnov goodness-of-fit K-S.

normal distribution and we do not use the mean and variance values of the fitted normal distributions, which are provided here for the sole indication purpose. Instead the meso-scale random fields are obtained from the data resulting from the stochastic homogenization as it will be discussed in Section 4.4.

Some spatial auto/cross-correlations (31) of the SSVEs' apparent elastic properties and volume fractions are presented in Fig. 17. For both SSVE lengths $l_{\mathrm{SSVE}}=10 \mu \mathrm{m}$ and $l_{\mathrm{SSVE}}=25 \mu \mathrm{m}$, these auto-correlations show that the apparent elastic properties and fiber volume fraction are only correlated when the two SSVEs are overlapping, i.e. when the distance $\|\tau\|$ is lower than $l_{\text {SSVE. }}$. Figs. $17(\mathrm{a})$ and $17(\mathrm{~b})$ indicate that for two SSVEs side by side, although they may share some common fibers, their apparent elastic properties and fiber volume fractions are not correlated. When considering the distance $\|\tau\|=0$, strong correlations between the elastic properties can be seen through their cross-correlations, Figs. 17(c) and 17(d). In particular, as expected, strong correlations exist between the elastic properties and the fiber volume fraction as between $E_{z}$ and $V_{f}$.

An important feature from this analysis, with a view to the resolution of macro-scale stochastic finite elements, is that the auto- and cross-correlations reach zero for adjacent SSVEs. In particular, for $l_{\mathrm{SSVE}}=25 \mu \mathrm{m}$, since the apparent elastic properties and fiber volume fraction follow nearly normal distributions, spatially uncorrelated properties means independent random variables. As a result the apparent elastic properties at the macro-scale can be treated as random variables instead of random fields.

\subsubsection{Effect of boundary conditions}

In the context of the computational homogenization method described in Section 4.1, the meso-scale finite element problem is solved by applying kinematically admissible boundary conditions. In this section, we study the effect of 


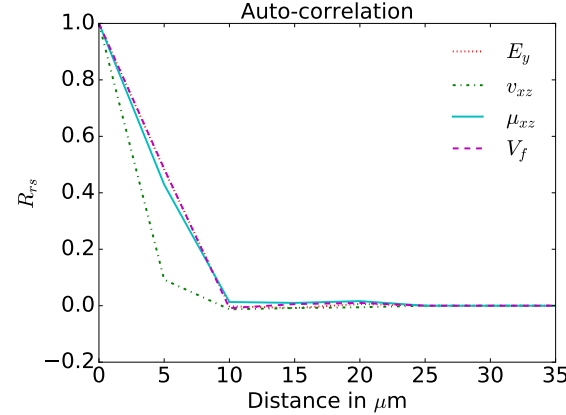

(a) $l_{\mathrm{SSVE}}=10 \mu \mathrm{m}$

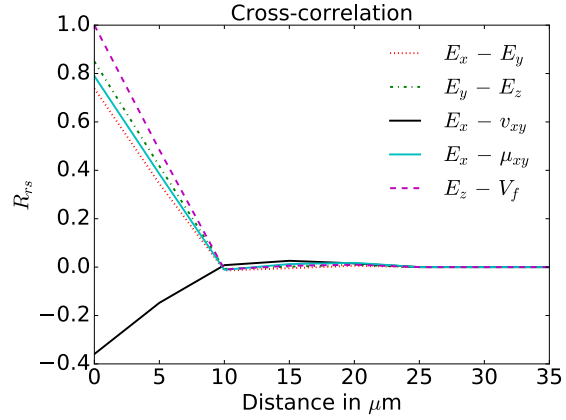

(c) $l_{\mathrm{SSVE}}=10 \mu \mathrm{m}$

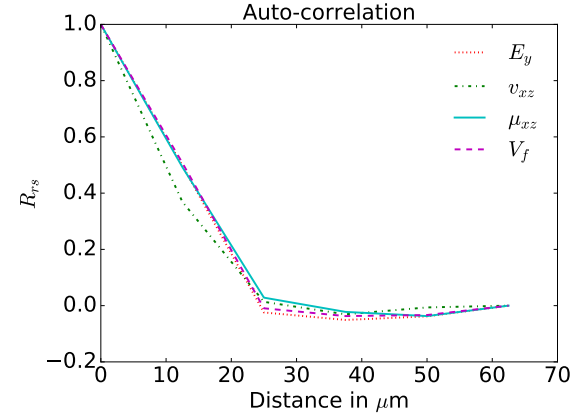

(b) $l_{\mathrm{SSVE}}=25 \mu \mathrm{m}$

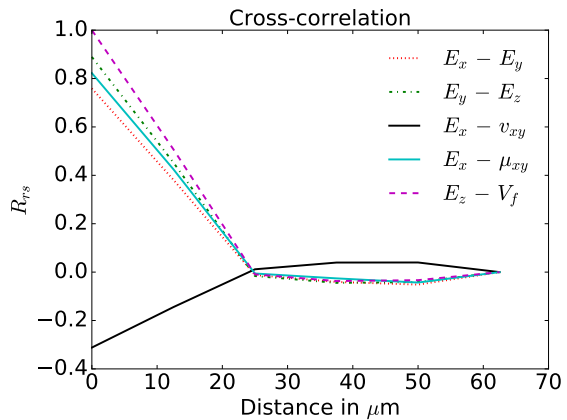

(d) $l_{\mathrm{SSVE}}=25 \mu \mathrm{m}$

Figure 17: The auto/cross-correlations of SSVEs' apparent elastic properties and fiber volume fraction: the left column refers to $l_{\mathrm{SSVE}}=10 \mu \mathrm{m}$ and the right column to $l_{\mathrm{SSVE}}=25 \mu \mathrm{m}$.

the boundary conditions on the apparent SSVEs' elastic properties by considering successively KUBCs, SUBCs and PBCs. In particular, we are interested in the properties in the $x-y$ plane and we focus the analysis on the apparent values of $E_{x}$.

The histograms of the apparent $E_{x}$ are displayed in Fig. 18 for the different boundary conditions, i.e. for KUBCs, SUBCs and PBCs. It appears that the distribution of $E_{x}$ obtained under different boundary conditions are quite different. Theoretically, for the same SVE, the apparent elastic property follows the property

$$
E_{x}^{\mathrm{SUBC}} \leq E_{x}^{\mathrm{PBC}} \leq E_{x}^{\mathrm{KUBC}},
$$

which is recovered on average in the histograms of $E_{x}$. With the increase of the SSVE size, from Fig. 18(a) to Fig. 18(b), we can see that the scatter in the apparent $E_{x}$ decreases, on the one hand for different SSVEs with the same applied boundary condition kind, and on the other hand when applying different boundary conditions on the same SSVE. Furthermore, for $l_{\mathrm{SSVE}}=10 \mu \mathrm{m}$, Fig. 18(a) shows that

$$
\min \left\{E_{x}^{\mathrm{SUBC}}\right\}=\min \left\{E_{x}^{\mathrm{PBC}}\right\}=\min \left\{E_{x}^{\mathrm{KUBC}}\right\}=E_{0}^{\mathrm{el}},
$$




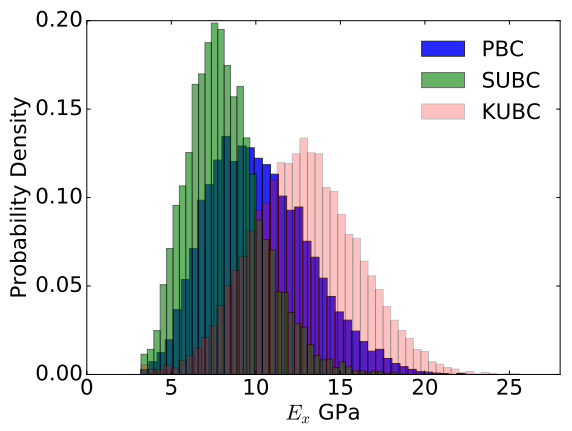

(a) $l_{\mathrm{SSVE}}=10 \mu \mathrm{m}$

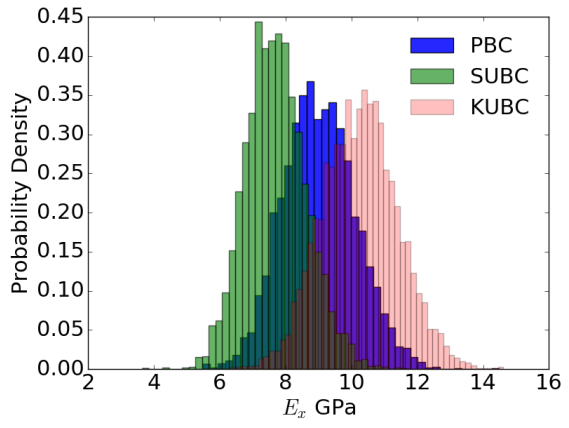

(b) $l_{\mathrm{SSVE}}=25 \mu \mathrm{m}$

Figure 18: The histograms of SSVEs' apparent $E_{x}$ for different boundary conditions: refers to $l_{\mathrm{SSVE}}=10 \mu \mathrm{m}$ and (b) to $l_{\mathrm{SSVE}}=25 \mu \mathrm{m}$.

which indicate a homogeneous SSVE. It turns out that the fiber volume fraction $V_{f}$ could be 0 for small SVEs. The effect of the boundary conditions reveals the uncertainty resulting from the in-homogeneously distribution of the fibers in the SVEs. The other source of uncertainty is the fiber volume fraction in the SVEs.

When considering the response of a small material window, in a real structural analysis, the apparent properties of the SSVE will barely be equal to those obtained from the computational homogenization under any of the three boundary condition kinds. In fact, the material window response is not unique and depends on the properties of the surrounding material:

- If the surrounding material is stiffer than in the SSVE, e.g. because the SSVE has a lower fiber volume fraction, strong constraints are applied on this SSVE and its response will be close to the results obtained under KUBCs.

- If the surrounding material is more compliant than in the SSVE, e.g. because the SSVE has a higher fiber volume fraction, weak constraints are applied on this SSVE and its response will be close to the results obtained under SUBCs.

- Finally, if the stiffness of the surrounding material is comparable to that of the SSVE, it response will be close to the results obtained under PBCs.

Therefore, for the SVEs with compliant apparent properties that can be seen at the left side of the histogram in Fig. 18, their in situ apparent properties are more likely to approach the properties obtained under KUBCs, and vice versa. Furthermore, because of the non uniqueness of the SSVEs' apparent properties, any given values are just approximated responses of the SSVE in a real structure. However, practically, the apparent elastic properties of the SSVEs obtained under PBCs provide a reasonable approximation of the elastic properties distributions as it can be seen in Fig. 18. 


\subsection{Apparent elastic properties of Big SVEs (BSVEs)}

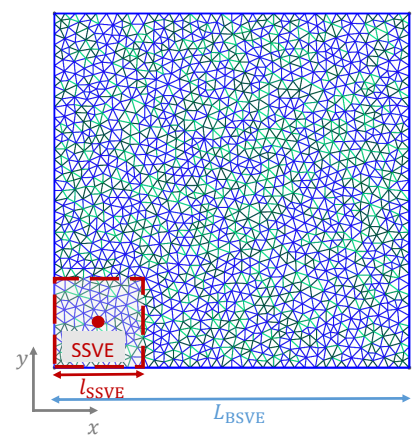

Figure 19: Illustration of the two-step computational homogenization: the mesh of a BSVE of length $L_{\mathrm{BSVE}}=100 \mu \mathrm{m}$ and the window corresponding to a SSVE of length $l_{\mathrm{SSVE}}=25 \mu \mathrm{m}$.

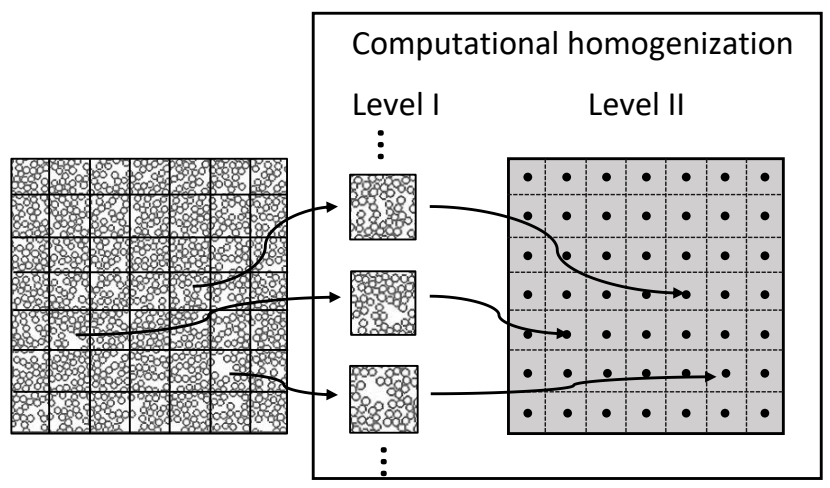

Figure 20: Illustration of the two-step computational homogenization considering nonoverlapping SSVEs.

In a real finite element analysis of composite structures, the computationally acceptable element size is around the millimeter. If we want to use the material properties extracted from the computational homogenization in this structural analysis, the SVEs, on which the homogenization is performed, should have a comparable size with the macro-scale element size of the structural analysis in order to capture the probabilistic characteristics.

Although performing a homogenization in linear elasticity on a single realization is achievable, in the context of stochastic homogenization and anticipating on the further developments, which include non-linear responses, the required computational time would become too important for practical applications. We thus study the possibility, in the context of linear elasticity in this work, to consider the stochastic response of the SSVEs to deduce the stochastic behavior of a larger one. 


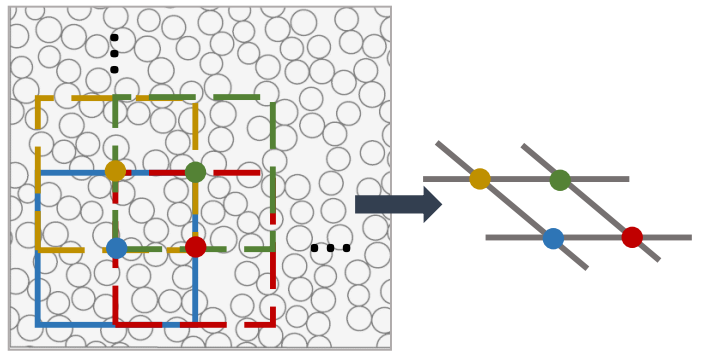

(a)

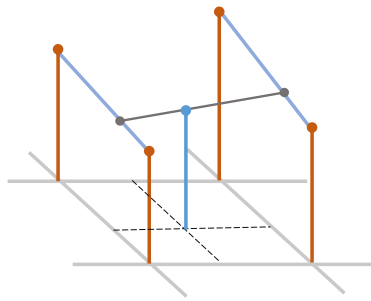

(b)

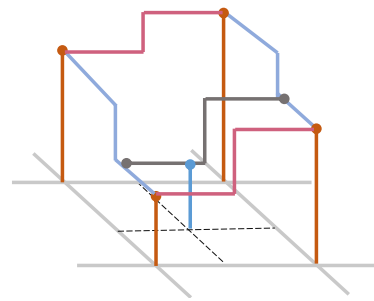

(c)

Figure 21: Illustration of the two-step computational homogenization considering overlapping SSVEs: (a) material property interpolation grid based on the homogenization of overlapping SSVEs, (b) bi-linear interpolation, and (c) nearest-neighbor interpolation.

\subsubsection{Two-step computational homogenization process for Big SVEs}

In order to reduce the computational time of the homogenization process of BSVEs, we apply a two-step homogenization as illustrated in Fig. 19. At the first level (level I), the BSVE is divided into a number of SSVEs, on which the computational homogenization is carried out in order to extract their homogenized elastic properties following the method described in Section 4.2. At the second level (level II), the homogenization is performed on a BSVE, whose finite element discretization, see Fig. 19, is finer than the size of the SSVEs as justified in the next Section 5, and whose material properties at different integration points correspond to the homogenized properties of the SSVEs.

In order to evaluate the material properties at the different integration points of the BSVEs, two approaches are developed:

1. By considering non-overlapping SSVEs (Fig. 20):

In this case denoted by "N-Intpl", during the homogenization at level II, the BSVE is divided into a number of small cells corresponding to nonoverlapping SSVEs, and each cell has a uniform material property, which is obtained from its corresponding SSVE.

2. By considering overlapping SSVEs (Fig.21(a)):

A 2D grid is built from the center points of the overlapping SSVEs, and to each of these points is associated the homogenized properties of their corresponding SSVE. At the homogenization of level II in the BSVE, the 
material properties of the integration points are obtained by interpolation from the 2D grid. In this section we consider two interpolation methods: bi-linear denoted by "IntplB" (Fig. 21(b)) and nearest-neighbor denoted by "IntplN" (Fig. 21(c)) interpolations.

\subsubsection{Verification of the two-step computational homogenization}

To verify the accuracy of the two-step computational homogenization, a BSVE is first generated and a direct one-step computational homogenization is applied on it in order to extract its apparent elastic properties, which will serve as the reference result. Then the two-step homogenization is performed on this BSVE by (i) dividing the BSVE into SSVEs whose apparent properties are computed by the level I computational homogenization, and (ii) using the SSVEs apparent properties to perform the level II computational homogenization on the BSVEs. Several sizes of BSVEs of length $L_{\mathrm{BSVE}}$, and of SSVEs of length $l_{\mathrm{SSVE}}$, are successively considered and both cases of overlapping and non-overlapping SVEs are studied.

In order to assess the accuracy of the method, the elastic apparent properties, $E_{x}, E_{y}, E_{z}, \nu_{x y}, \nu_{x z}, \nu_{y z}, \mu_{x y}, \mu_{x z}$ and $\mu_{y z}$, of the BSVEs obtained from direct and two-step computational homogenization resolutions are compared, and the relative error is defined as

$$
\text { err. }=(\cdot \mathrm{TS}-\cdot \operatorname{dir}) / \cdot \operatorname{dir},
$$

where $\cdot$ is one of the nine material parameters $\left(E_{x}, E_{y}, E_{z}, \nu_{x y}, \nu_{x z}, \nu_{y z}, \mu_{x y}\right.$, $\mu_{x z}$, and $\left.\mu_{y z}\right)$, $\cdot$ dir is obtained from direct homogenization of the BSVE and $\cdot_{\mathrm{TS}}$ from the two-step process.

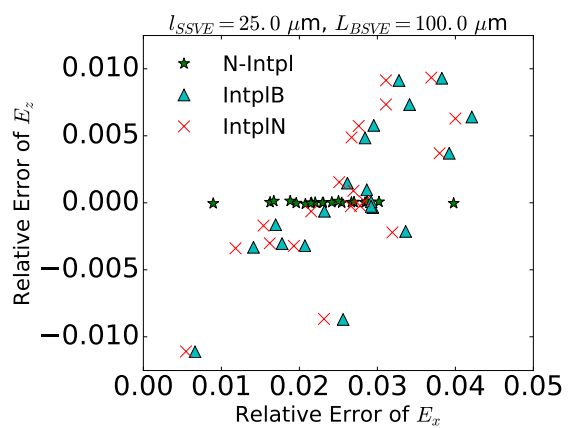

Figure 22: Relative errors of BSVEs' apparent $E_{x}$ and $E_{z}$ for the different 2-step homogenization methods.

Errors related to the interpolation methods. The three methods described in Section 4.3.1, i.e. "N-Intpl" using non-overlapping SSVEs, "IntplB" using overlapping SSVEs with bi-linear interpolation, and "IntplN" using overlapping SSVEs with nearest neighbor interpolation, are successively applied with SSVEs of length $l_{\mathrm{SSVE}}=25 \mu \mathrm{m}$ and on BSVEs of length $L_{\mathrm{BSVE}}=100 \mu \mathrm{m} .20 \mathrm{BSVE}$ 
realizations are considered. In this section, Periodical Boundary Conditions (PBCs) are adopted during the computational homogenization processes.

The relative errors on the BSVE apparent $E_{x}$ and $E_{z}$, which are defined in Eq. (35), are reported in Fig. 22, where it can be seen that, all over all, the "NIntpl" method gives more accurate results for $E_{x}$ and $E_{z}$ than when considering overlapping SSVEs. In the latter case, the two interpolation methods "IntplB" and "IntplN" lead to comparable errors for both $E_{x}$ and $E_{z}$. Therefore in what follows we only consider the interpolation "IntplB" type when using overlapping SSVEs.

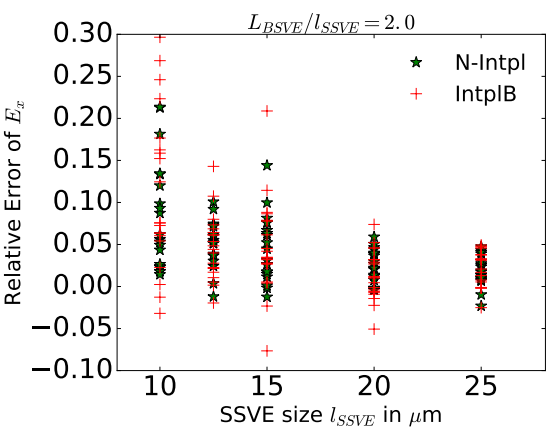

(a)

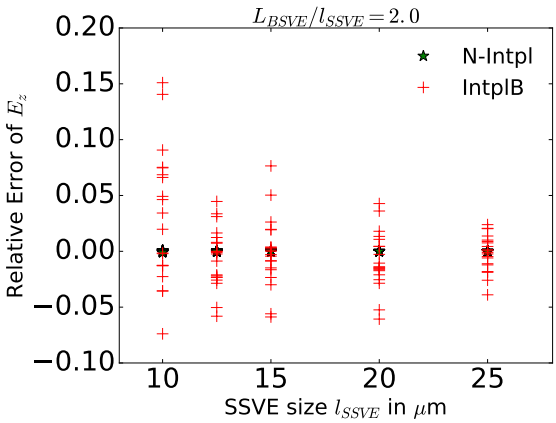

(c)

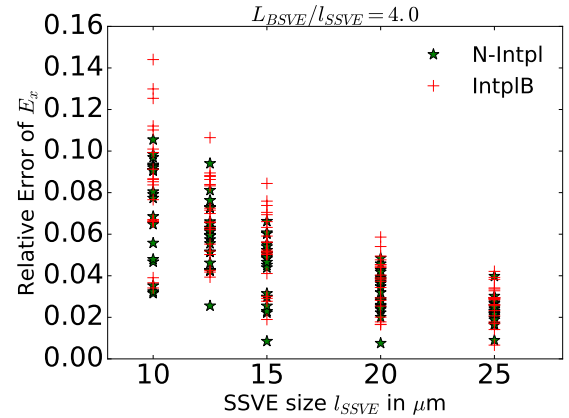

(b)

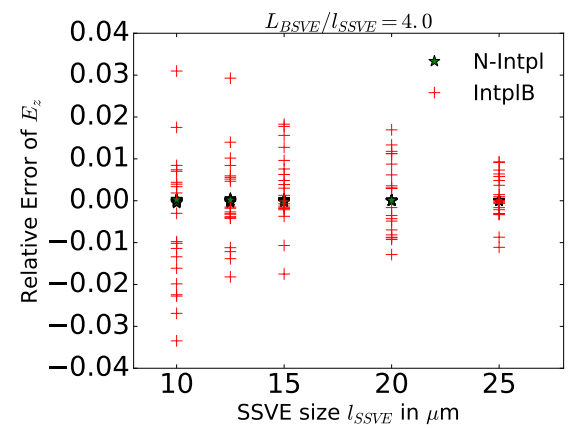

(d)

Figure 23: Relative errors of BSVEs' apparent $E_{x}$ and $E_{z}$ for the different ratios of BSVEs and SSVEs lengths $L_{\mathrm{BSVE}} / l_{\mathrm{SSVE}}$ : the left column refers to $l_{\mathrm{SSVE}} / L_{\mathrm{BSVE}}=2$ and the right column to $l_{\mathrm{SSVE}} / L_{\mathrm{BSVE}}=4$.

More comparisons between "N-Intpl" and "IntplB" methods are carried out by considering different ratios between the SSVEs and BSVEs lengths. The studied SSVEs lengths are successively $l_{\mathrm{SSVE}}=10,12.5,15,20$ and $25 \mu \mathrm{m}$, and the corresponding $L_{\mathrm{BSVE}}$ are set to be successively $2.0 \times$ and $4.0 \times l_{\mathrm{SSVE}}$. For each pair $\left(l_{\mathrm{SSVE}}, L_{\mathrm{BSVE}}\right) 20$ realizations of BSVEs are considered.

The relative errors on the BSVE apparent properties, which are defined in Eq. (35), are reported in Figs. 23(a) and 23(b) for the apparent $E_{x}$, and in Figs. $23(\mathrm{c})$ and $23(\mathrm{~d})$ for the apparent $E_{z}$. As for the previous study, the "N-Intpl" 
method always leads to an accurate apparent $E_{z}$ of BSVE. With the increase of $l_{\mathrm{SSVE}}$, the errors on the apparent $E_{x}$ of the BSVEs obtained by the "N-Intpl" and "IntplB" methods become comparable. Therefore, for the further analyses we consider only the "N-Intpl" method.

Errors related to the BSVEs length $L_{B S V E}$ and to the SSVEs length $l_{S S V E}$. Using the "N-Intpl" method, we deepen the study related to the effect of the BSVEs and SSVEs lengths on the homogenization error. We consider the two SSVEs lengths $l_{\mathrm{SSVE}}=10$ and $25 \mu \mathrm{m}$. For the SSVEs length of $l_{\mathrm{SSVE}}=10 \mu \mathrm{m}$ $\left(l_{\mathrm{SSVE}}=25 \mu \mathrm{m}\right)$, the BSVEs length $L_{\mathrm{BSVE}}$ is successively set to be $2 \times, 3 \times$, $4 \times$, and $5 \times$ (respectively $2 \times, 3 \times$, and $4 \times$ ) the SSVEs length $l_{\text {SSVE. }}$ As in the previous analysis, for each pair of $\left(l_{\mathrm{SSVE}}, L_{\mathrm{BSVE}}\right) 20 \mathrm{BSVE}$ realizations are considered.

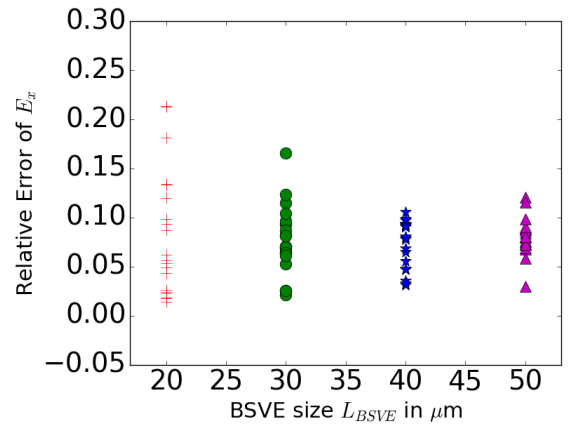

(a) $l_{\mathrm{SSVE}}=10 \mu \mathrm{m}$

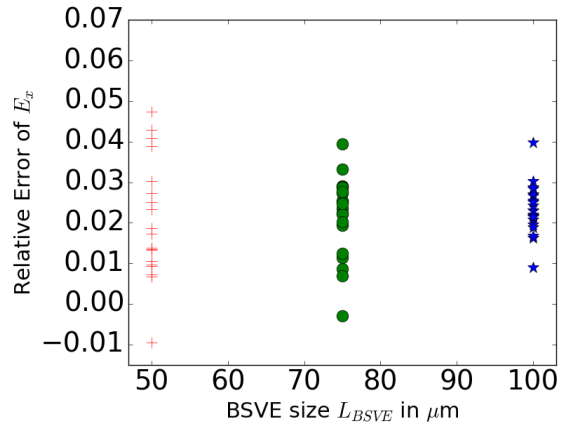

(b) $l_{\mathrm{SSVE}}=25 \mu \mathrm{m}$

Figure 24: Relative errors of BSVEs' apparent $E_{x}$ for different BSVEs lengths $L_{\mathrm{BSVE}}$ : (a) $l_{\mathrm{SSVE}}=10 \mu \mathrm{m}$ and (b) $l_{\mathrm{SSVE}}=25 \mu \mathrm{m}$.

Since the relative error on $E_{x}$ has been shown to be the higher in the previous analysis, only this apparent elastic properties is studied. Figure 24 shows that the errors on $E_{x}$ obtained through the two-step homogenization process decrease with the increase of $L_{\mathrm{BSVE}}$. When comparing Figs. 24(a) and 24(b) for $L_{\mathrm{BSVE}}=$ $50 \mu \mathrm{m}$, it can be seen that the increase of $l_{\mathrm{SSVE}}$ also leads to a decrease of the errors. Moreover, the combined effects of $L_{\mathrm{BSVE}}$ and $l_{\mathrm{SSVE}}$ on the accuracy of the two-step process can also be studied from Fig. 23 and explained as follows:

- With the increase of $l_{\text {SSVE }}$, the scatter in the SSVEs' apparent elastic properties decreases, leading to a reduced properties contrast between the neighboring SSVEs. As a result, the applied PBCs on SSVEs become closer to the real constraints on the volume elements in the larger window.

- When a BSVE includes more SSVEs, the errors, resulting from the use of approximated properties at the integration point of the BSVEs, become less prominent in the result of BSVE. 
Errors related to the boundary conditions. When increasing the size of the BSVEs, the scatter in their elastic properties decreases. An adequate stochastic homogenization process should ensure that the scatter in the BSVEs' elastic properties is not covered by the error introduced by the homogenization process. In the presented two-step homogenization process, up to now the PBCs were used to approximate the real boundary conditions on the SSVEs as discussed in Section 4.2.2.

In order to verify the accuracy of applying PBC on the SSVEs, direct computational homogenization resolutions are carried out on 20 BSVEs of size $100 \times 100 \mu \mathrm{m}^{2}$ successively using KUBCs and PBCs in order to provide reference results. The two-step homogenization process is then considered for the same 20 BSVE realizations. In the two-step process, KUBCs, PBCs, and SUBCs are successively applied on the SSVEs of size of $25 \times 25 \mu \mathrm{m}^{2}$. The non-overlapping "N-Intpl" method is used during the level II homogenization to compute the apparent properties of the BSVEs.

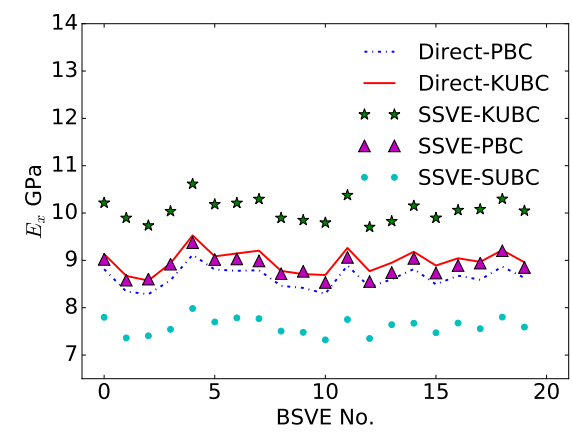

(a) PBCs on BSVEs

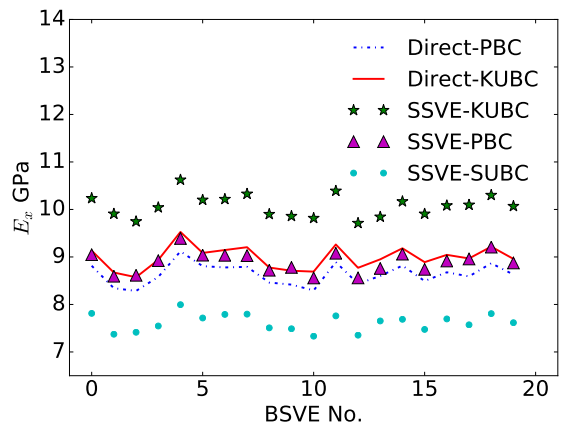

(b) KUBCs on BSVEs

Figure 25: Apparent $E_{x}$ of different BSVE realizations obtained through the two-step process for different boundary conditions applied on the SSVEs when considering (a) PBCs and (b) KUBCs on the BSVEs: "Direct-PBC" and "Direct-KUBC" refer to the results obtained through direct computational homogenization on BSVEs; "SSVE-X" refer to the boundary condition type " $\mathrm{X}$ " applied on the SSVEs during the two-step process.

The comparison between the BSVEs' apparent $E_{x}$ obtained through the direct homogenization and through the two-step homogenization processes is presented in Fig. 25. Using PBCs or KUBCs on the BSVEs for the twostep process does not significantly change the BSVEs' apparent properties: the apparent $E_{x}$ obtained using KUBCs on the BSVEs (Fig. 25(b)) are just slightly higher -within 0.3\% - than those obtained using PBCs on the BSVEs (Fig. 25(a)). However, the effect of the boundary condition types applied on the SSVEs is obvious. On the one hand, when applying KUBCs (SUBCs) on the SSVEs, the two-step process leads to unacceptable stiff (respectively compliant) apparent properties of the BSVEs. On the other hand, when applying PBCs on the SSVEs, the two-step homogenization process leads to results in between the apparent elastic properties obtained by the direct homogenization of the BSVEs with KUBCs and PBCs. This means that PBC applied on the SSVEs 
are representative to the real constraints on this material windows within the BSVEs.

\subsubsection{Conclusion on the two-step homogenization process}

According to the error studies conducted in Section 4.3.2, it can be concluded that for sufficiently high sizes of SSVEs $\left(l_{\mathrm{SSVE}} \geq 25.0 \mu \mathrm{m}\right.$ in this work), when applying PBCs during the SSVEs homogenization, the error induces by the twostep homogenization process is within the error introduced by the boundary conditions.

Moreover, as shown in Fig. 25, the difference in the apparent elastic properties between the two-step process and the direct homogenization is of the same order for the different BSVE realizations, and is lower than the scatter between the apparent properties of the different BSVEs. The two-step method can thus be applied during a stochastic homogenization process to define the apparent properties of BSVEs.

\subsection{Generation of apparent meso-scale properties}

The study of Section 4.3.2 shows that a better accuracy is obtained by using the properties of non-overlapping SSVEs during the two-step homogenization process. Moreover, it has been shown in Section 4.2 that the elastic properties of two adjacent SSVEs are uncorrelated random vectors. Finally, when the SSVEs size increases, it has also been shown that their elastic properties get closer to a normal distribution. Hence, we can assume that the elastic properties of two SSVEs without overlap are independent random vectors. This means that a random vector field can be created by generating a series of independent random vectors, which represent the random apparent properties of the SSVEs. The obtained random vector field exhibits a unit-correlation for two points lying in the same SSVE and a zero-correlation for two points lying in different SSVEs. These random vectors can thus be used as material properties in a stochastic finite element analysis.

Using apparent properties obtained from a given amount of SSVE realizations, a random vector generator can thus be constructed using the method of copula [54] presented in Section 3.1 and the data-driven sampling method [63]. If a random vector field was required, the spectral representation method, which has been used in [48] to generate Gaussian random vector fields and in [33] to generate non-Gaussian random vector fields, can also be used. In this work, the apparent elastic properties, are random vectors generated using the copula method [54] with as input the nine elastic properties obtained by the stochastic homogenization performed on the SSVEs. Since we are dealing with 9 components, the copula method requires a high number of samples to be accurate. A principal component analysis is thus combined to an order reduction to reach 5 variables and 10000 samples are thus enough for the copula method to converge.

In order to evaluate the computational efficiency of the stochastic homogenization method, stochastic apparent properties of a BSVE are successively obtained through three different processes: 
- In the first process, direct computational homogenizations, i.e. discretizing explicitly the volume element up to the micro-structure level, are carried out on BSVEs.

- In the second process, the two-step homogenization, discussed in Section 4.3.1, is applied by applying computational homogenization on successively the SSVEs (at level I) and the BSVEs (at level II).

- In the third process, the two-step homogenization is performed in a different ways. From a set of SSVE realizations, a random vector generator is constructed using the copula method. The level II homogenization is thus performed using random apparent material properties, associated to the SSVEs, obtained by the generator, which avoids to conduct the step I homogenization.

Table 1: Computational efficiency of the stochastic homogenization methods: the reported CPU time corresponds to the time requires to obtain the homogenized properties of one BSVE realization.

\begin{tabular}{lll}
\hline $\begin{array}{l}\text { Homogenization method } \\
\text { of BSVE }\end{array}$ & $\begin{array}{l}\text { SSVE/BSVE } \\
\text { mesh sizes }[\mu \mathrm{m}]\end{array}$ & CPU times $[\mathrm{s}]$ \\
\hline $\begin{array}{l}\text { One-step } \\
\text { Two-step homogenization }\end{array}$ & $-/ 1.5$ & $\approx 1250$ \\
$\begin{array}{l}\text { Random vector generator \& } \\
\text { computational homogenization }\end{array}$ & $-/ 5$ & $25 \times 3.6+4=93$ \\
\hline
\end{tabular}

A volume element of $125 \times 125 \mu \mathrm{m}^{2}$ is used here to test the efficiency of the three proposed processes. When considering the second and third processes, this volume element corresponds to the BSVE, and is partitioned into 25 SSVEs of size $25 \times 25 \mu \mathrm{m}^{2}$. The CPU times required to extract the apparent properties of a $125 \times 125 \mu \mathrm{m}^{2}$ BSVE are presented in Table 1 for the three different processes. In Table 1, it can been seen that performing the two-step homogenization is computationally efficient in particular when using a random vector generator. In fact, the random vector generators rely on the fact that only a reduced set of SVE realizations is needed to generate as many samples as required. Of course obtaining these realizations and building the generator requires a CPU time, but this corresponds to a one-time off-line computation whose time was not reported in Table 1. Random vector generators are commonly used in the stochastic finite element analyzes, and data-driven random variables generator becomes popular along with the huge improvement of the ability of computers.

Finally the apparent properties of a set of BSVE realizations can also be used to build another random vector generator in order to be able to gener- 
ate stochastic apparent properties for the macro-scale stochastic finite element analyzes.

\section{Application to stochastic finite element analyzes}

In a stochastic finite element analysis, two kinds of discretization need to be carried out. The first one is the discretization of a continuous structure into finite elements. The second one is the discretization of the random material properties field, which provides the random properties at each integration point of the finite elements. In this work, the random vector generator described in Section 4.4 is used to generate stochastic apparent elastic properties, which are in turn used as input stochastic finite element analyzes. To ensure the convergence of the stochastic finite element analysis, the characteristic finite element size needs to be smaller than the correlation length of the applied random field. Therefore, the finite element size needs to be lower than the size of the SVEs used to create the random vector field. This justifies the use of finer mesh in the BSVEs than the size of the SSVEs in Section 4.3.1.

In this Section, we first illustrate how to apply the methodology on a simple ply tensile test. However since we consider only elasticity, tensile tests yield a non-uniform stress distribution within the ply but the ply response remains deterministic because statistical representativity is recovered at the loading length scale. Therefore we then perform a stochastic finite element simulation on a ply under bending yielding a distribution in the bending response and in the maximum bending stress of the ply.

\subsection{Stochastic tensile samples}

As an illustration example we perform here below a simple stochastic finite element analysis of a ply cross-section using the material properties random vectors.

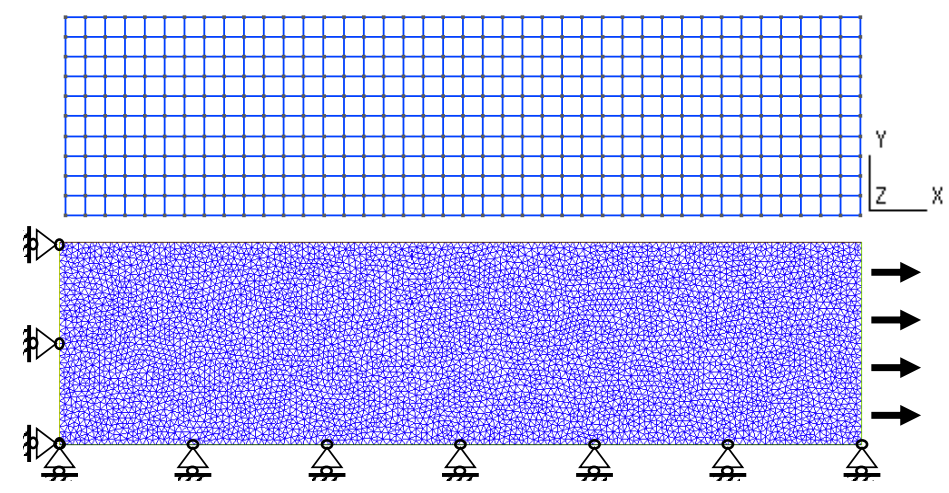

Figure 26: Tensile stochastic finite element analyzes: discretizations of the random field (top) and of the finite element structure (bottom). 
A composite cross-section of $1000 \times 250 \mu \mathrm{m}^{2}$ is loaded in the transverse direction with plane strain condition along the $z$-direction and plane stress condition along the $y$-direction. An illustration of the detailed micro-structure corresponding to a ply of the same width can be found in Fig. 31(a). The random vector generator described in Section 4.4 is used to generate stochastic apparent elastic properties corresponding to SSVEs of sizes $25 \times 25 \mu \times 1 \mathrm{~m}^{3}$, which are in turn used as input stochastic finite element analyzes. The discretizations of the random field and of the finite element are shown in Fig. 26 along with the boundary and loading conditions that are applied on the structure.

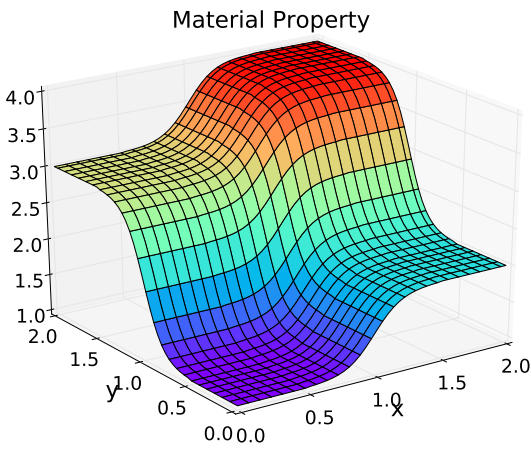

(a)

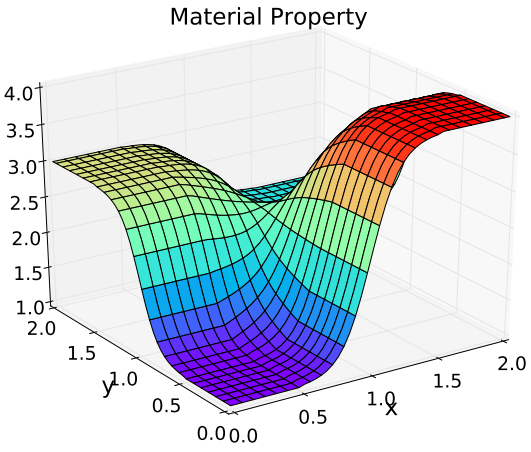

(b)

Figure 27: Examples of apparent properties (the properties are not realistic but chosen with a high contrast for illustration purpose) interpolated at the boundaries of the random field grid using a smooth step function The boundaries of the random field grid correspond to the coordinates $x=1$ or $y=1$.

The random field of the material properties is discretized into squares of $25 \times$ $25 \mu \mathrm{m}^{2}$. Therefore, the transition of the material properties in the random field discretization follows a step function. However, a strong contrast of material properties could exist at the boundary of the material properties random field discretization, which would introduce unexpected stress concentrations in the finite element resolution. In order to avoid this artificial stress concentration, smooth-step functions, see Fig. 27, are used to describe the transition of material properties at the internal boundaries of the random field discretization.

Four realizations of the $1000 \times 250 \mu \mathrm{m}^{2}$ samples are put under transverse loading to reach a macro-strain of 0.006 . The homogenized stress distribution $\sigma_{\mathrm{M} x x}$ in the samples are presented in Fig. 28 for the four realizations. The resulting average macro-stress (the resultant force along the $x$-direction divided by the transverse section) varies from $53.5 \mathrm{MPa}$ to $54.4 \mathrm{MPa}$ for the four realizations. However, the maximum homogenized stresses $\sigma_{\mathrm{M} x x}$-which is not the maximum stress reached in the different phases of the composite materialreached in the different four realizations are $63.4,65.8,67.3$ and $66.8 \mathrm{MPa}$, respectively. To emphasize the uncertainty at the ply level, we consider in the next application a ply under bending. 


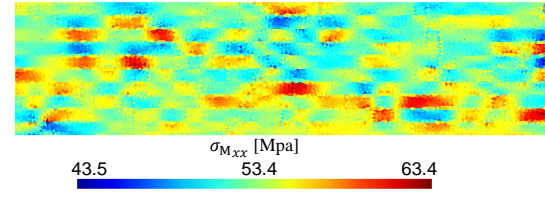

(a)

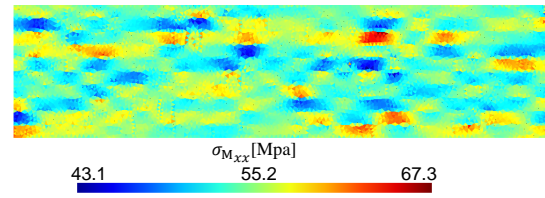

(c)

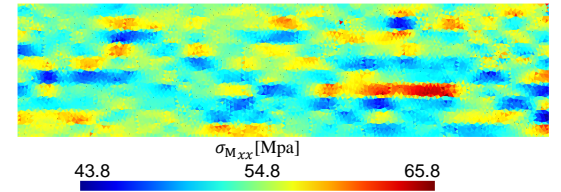

(b)

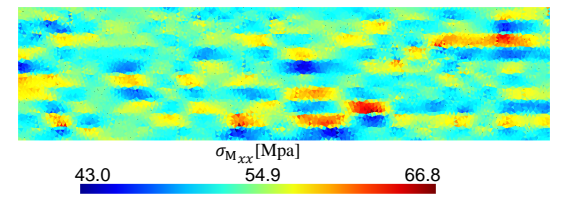

(d)

Figure 28: Meso-scale stress distributions in four $1000 \times 250 \mu \mathrm{m}^{2}$ realizations under transverse loading.

\subsection{Stochastic bending samples}

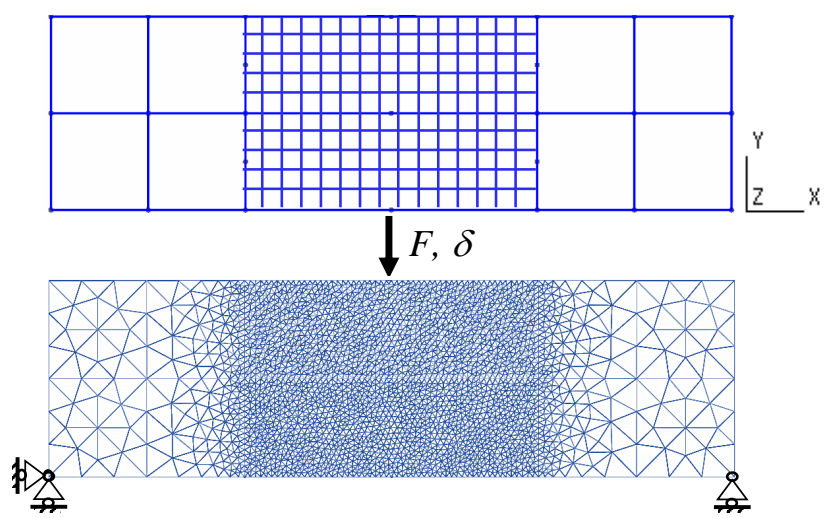

Figure 29: Bending stochastic finite element analyzes: discretizations of the random field (top) and of the finite element structure (bottom).

A composite cross-section of $875 \times 250 \mu \mathrm{m}^{2}$ is under three-point bending, see Fig. 29. A displacement $\delta$ is applied at the upper mid-length resulting in a loading force $F$ along the $y$-direction. The sample is under plane-strain condition along the $z$-direction (fiber direction). We study the effect of material uncertainties on the loading force $F$ and the maximum tensile stress $\sigma_{\mathrm{M}_{x x} x}$.

The discretizations of the random field and of the finite elements are shown, along with the boundary and loading conditions, in Fig. 29. The random field at the two ends of the sample is discretized into eight domains of size $125 \times 125 \mu \mathrm{m}^{2}$. In the middle part of the sample, the random field of material properties is discretized into squares of $25 \times 25 \mu \mathrm{m}^{2}$. In this last part, the random vector generator described in Section 4.4 is used to generate stochastic 
apparent elastic properties corresponding to SSVEs of sizes $25 \times 25 \mu \mathrm{m}^{3}$. In the coarser part, the random apparent properties of the $125 \times 125 \mu \mathrm{m}^{2}$ BSVEs are obtained through the two-step homogenization using random vectors for the SSVEs material properties, i.e. using the third process described in Section 4.4. The newly obtained random vector field exhibits a unit-correlation for two points lying in the same BSVE and a zero-correlation for two points lying in different BSVEs. As a result the mesh of the structure can be coarser at the ply extremities as illustrated in Fig. 29 and finer in the middle of the sample to ensure an accurate resolution, while the finite element size remains lower than the size of SVEs everywhere. 800 bending realizations are computed to ensure the convergence of the ply behavior distribution.

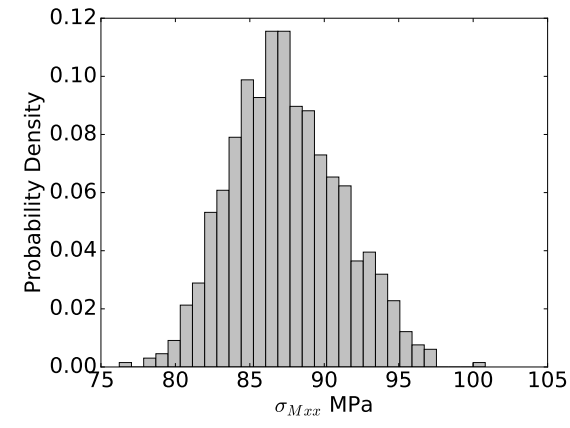

(a) $\mathbb{E}=87.44 \mathrm{MPa}, \sigma=3.70 \mathrm{MPa}$

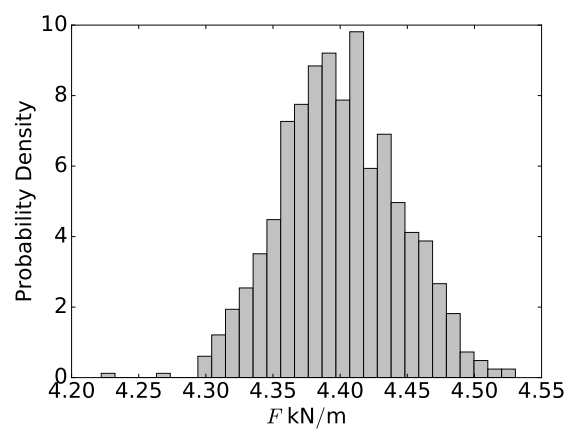

(b) $\mathbb{E}=4.400 \mathrm{kN} \cdot \mathrm{m}^{-1}, \sigma=0.044 \mathrm{kN} \cdot \mathrm{m}^{-1}$

Figure 30: The histograms of the stochastic bending test for a displacement $\delta=8.0 \mu \mathrm{m}$ : (a) the maximum tensile stress $\sigma_{\mathrm{M}_{x x}}$ reached in each sample and (b) the applied loading $F$ per unit thickness; the mean value $\mathbb{E}$ and standard deviation $\sigma$ are also reported.

The histograms of the homogenized tensile stress $\sigma_{\mathrm{M} x x}$ at the mid-length lower face -which is the maximum homogenized tensile stress but which is not the maximum stress reached in the different phases of the composite materialreached in each sample and the applied loading $F$ per unit thickness are presented in Fig. 30 for a maximum displacement $\delta=8.0 \mu \mathrm{m}$ at the loading point. Uncertainty in the results can be seen in the histograms, especially for the reached maximum tensile stress $\sigma_{\mathrm{M} x x}$, which shows more scatter and explains the discrepancy observed during failure analysis of composites.

However, we need to note that this maximum homogenized tensile stress cannot be used directly for the failure analysis. Indeed, this does not give a direct information on the stress in the phases; the latter could be obtained using a localization step. Moreover, the discrepancy in the maximum homogenized tensile stress also depends on the size of the SVEs considered to define the random vector of apparent properties. Since all the analyzes are based on a stochastic multi-scale process, the critical failure stress at each material point is an uncertain value too, which could be extracted in a probabilistic way from SVEs simulations as in $[49,50]$. In particular, probabilistic macro-failure criteria, such as Drucker Prager, have been extracted from SVE realizations in [50]. 


\section{Conclusions}

In order to carry out a stochastic multiscale analysis for UD fiber reinforced composites, two important issues are studied in this work. First, based on the SEM images of UD fiber reinforced composites' cross-section, the microstructure is statistically characterized. Using the obtained empirical statistical descriptors and their correlation information, a micro-structure generator is constructed to provide virtual micro-samples for numerical analyzes. Although in this work, only the random distribution of fibers is considered, micro-defects, such as micro-voids and micro-cracks, can also be accounted for if their descriptions, which include the geometries and distributions, are available.

Second, a multi-level computational homogenization is presented to extract the meso-response of large SVEs in an efficient way. The effects of different applied boundary conditions during the computational homogenization are studied and it was shown that periodic boundary condition is an accurate choice for the multi-level computational homogenization. Since a large gap exists between the micro-structure scale and the structure scale of UD composites, the presented multi-level process makes the stochastic multiscale analysis computationally affordable.

For illustration purpose of the method efficiency, two stochastic multiscale finite element analyzes are performed on relatively large random samples, leading to a nonuniform homogenized stress distribution and to an uncertain ply behavior as the results of a non-uniform micro-structure distribution.

Although the work is limited to elastic analysis, the presented procedure has the potential to be extended to the nonlinear regime and failure analysis. Nevertheless, combining computational homogenization on SVEs to MC simulations can lead to a prohibitive costs in the context of non-linear materials. In [64], the stochastic homogenization of UD composite cell was achieved by using a modified version of the meso-scale stochastic finite element method (SFEM), leading to a more efficient solution. Relying on the use of SVEs, stochastic multiscale analyzes were developed to account for fine-scale material properties as random variables -and random fields in particular cases- using an order reduction method combined to an asymptotic homogenization [65]. In the context of finite elasticity, a Reduced Order Model (ROM) was built from the resolution of SVEs, which are called composite material elementary cells, by defining a meso-scale potential capturing the uncertainties related to the fibers geometry/distribution in composites [66, 67]. In a more general way, the use of Reduced Order Models (ROMs) in data-driven non-linear stochastic analyzes is discussed in [68]. Therefore, in order to extend this work to non-linear analyzes, the definition of an adequate ROM will be investigated in the future to ensure its computational efficiency.

\section{Acknowledgment}

The research has been funded by the Walloon Region under the agreement no 1410246-STOMMMAC (CT-INT 2013-03-28) in the context of the M-ERA.NET 
Joint Call 2014.

SEM analyzes were performed with the help of Prof. Dr. Valéria Mertinger, head of institute, University of Miskolc, Faculty of Materials Science and Engineering, Department of Physical Metallurgy and Metalforming, Hungary.

\section{Appendix A. Generation of inhomogeneous micro-structures}

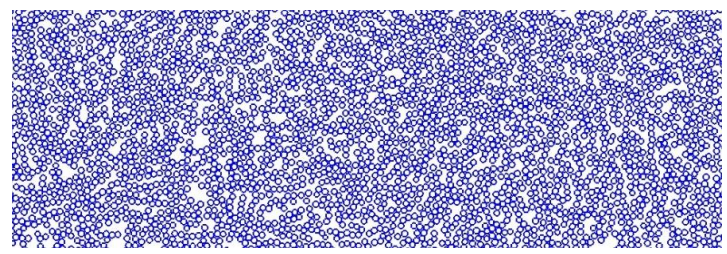

(a)

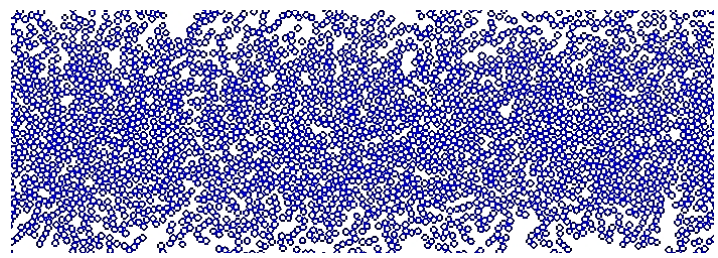

(b)

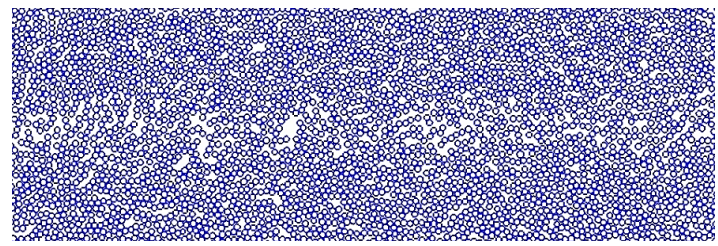

(c)

Figure A.31: Generated inhomogeneous micro-structures for a cross-section of $700 \times 250 \mu \mathrm{m}^{2}$ with (a) $\alpha=1.0$, (b) $\alpha(\boldsymbol{x})$ increasing from the window center line to the upper and lower boundaries, and (c) $\alpha(\boldsymbol{x})$ decreasing from the window center line to the upper and lower boundaries.

In this micro-structure generation procedure, $\vartheta_{1 \mathrm{st}}$ and $\Delta \vartheta$ are still considered as random variables and we consider the spatially non-stationary random variable $\tilde{d}_{1 \text { st }}$ and $\Delta \tilde{d}$ to replace $d_{1 \text { st }}$ and $\Delta d$, respectively. As a way of illustration, the non-stationary random variable is expressed as

$$
\tilde{d}_{1 \mathrm{st}}(\boldsymbol{x})=\alpha(\boldsymbol{x}) d_{1 \mathrm{st}} \quad \text { and } \quad \Delta \tilde{d}(\boldsymbol{x})=\alpha(\boldsymbol{x}) \Delta d,
$$

where $\alpha(\boldsymbol{x})$ is a coefficient function depending on the locations of central fibers, and $d_{1 \text { st }}$ and $\Delta d$ are the former random variables. The generated micro-structures are presented in Fig. A.31 for different functions $\alpha$, leading to different spatial distributions. 


\section{Appendix B. Resolution of the constrained meso-scale BVP}

The set of equations (28) is solved by the multiplier elimination method with $\boldsymbol{\lambda}=\mathbf{C}^{T}\left(\mathbf{C C}^{T}\right)^{-1} \mathbf{K}_{\mathrm{m}} \mathbf{u}_{\mathrm{m}}$, allowing to rewritten the set of equations (28) under the residual form

$$
\left\{\begin{array}{l}
\mathbf{r}_{\mathrm{m}}=\mathbf{K}_{\mathrm{m}} \mathbf{u}_{\mathrm{m}}-\mathbf{C}^{T} \boldsymbol{\lambda}=\mathbf{Q}^{T} \mathbf{K}_{\mathrm{m}} \mathbf{u}_{\mathrm{m}}=0, \text { and } \\
\mathbf{r}_{\mathrm{c}}=\mathbf{C} \mathbf{u}_{\mathrm{m}}-\mathbf{S} \mathcal{E}_{\mathrm{M}}=0,
\end{array}\right.
$$

with $\mathbf{Q}=\mathbf{I}-\mathbf{C}^{T}\left(\mathbf{C C}^{T}\right)^{-1} \mathbf{C}$, see details in [58]. Linearizing the system (B.1) with respect to both the displacement field and the macro-scale kinematic field leads to

$$
\left\{\begin{array}{l}
\mathbf{r}_{\mathrm{m}}+\mathbf{Q}^{T} \mathbf{K}_{\mathrm{m}} \mathbf{Q} \delta \mathbf{u}_{\mathrm{m}}-\mathbf{Q}^{T} \mathbf{K}_{\mathrm{m}} \mathbf{C}^{T}\left(\mathbf{C C}^{T}\right)^{-1}\left[\mathbf{r}_{\mathrm{c}}-\mathbf{S} \delta \mathcal{E}_{\mathrm{M}}\right]=0, \text { and } \\
\mathbf{r}_{\mathrm{c}}+\mathbf{C} \delta \mathbf{u}_{\mathrm{m}}-\mathbf{S} \delta \mathcal{E}_{\mathrm{M}}=0
\end{array}\right.
$$

The solution of the meso-scale BVP is obtained by considering $\delta \mathcal{E}_{\mathrm{M}}=0$ in (B.2) and the solution reads

$$
\delta \mathbf{u}_{\mathrm{m}}=-\tilde{\mathbf{K}}_{\mathrm{m}}^{-1} \tilde{\mathbf{r}}_{\mathrm{m}}
$$

with

$$
\left\{\begin{array}{l}
\tilde{\mathbf{K}}_{\mathrm{m}}=\mathbf{C}^{T} \mathbf{C}+\mathbf{Q}^{T} \mathbf{K}_{\mathrm{m}} \mathbf{Q}, \text { and } \\
\tilde{\mathbf{r}}_{\mathrm{m}}=\mathbf{r}_{\mathrm{m}}+\left(\mathbf{C}^{T}-\mathbf{Q}^{T} \mathbf{K}_{\mathrm{m}} \mathbf{C}^{T}\left(\mathbf{C} \mathbf{C}^{T}\right)^{-1}\right) \mathbf{r}_{\mathrm{c}}
\end{array}\right.
$$

The homogenized stress tensor (15) can be expressed under the vector form $\mathcal{S}_{\mathrm{M}}$ as

$$
\mathcal{S}_{\mathrm{M}}=\left(\frac{1}{V(\omega)} \bigwedge_{\omega^{e}} \int_{\omega^{e}} \mathcal{C}_{\mathrm{m}} \mathbf{B}^{e} \mathrm{~d} V\right) \mathbf{u}_{\mathrm{m}}=\mathbf{D} \mathbf{u}_{\mathrm{m}} .
$$

The apparent elasticity tensor in the matrix form $\mathcal{C}_{\mathrm{M}}$ is then obtained by derivation of (B.5) with respect to the macro-scale kinematic variable $\mathcal{E}_{\mathrm{M}}$. In order to evaluate $\frac{\partial \mathbf{u}_{\mathrm{m}}}{\partial \mathcal{E}_{\mathrm{M}}}$, we set $\mathbf{r}_{\mathrm{m}}=0$ and $\mathbf{r}_{\mathrm{c}}=0$ in the system (B.2), which results in

$$
\mathcal{C}_{\mathrm{M}}=\mathbf{D} \tilde{\mathbf{K}}_{\mathrm{m}}^{-1}\left(\mathbf{C}^{T}-\mathbf{Q}^{T} \mathbf{K}_{\mathrm{m}} \mathbf{C}^{T}\left(\mathbf{C} \mathbf{C}^{T}\right)^{-1}\right) \mathbf{S} .
$$

The details of the method can be found in [58]. The advantage of the method is that the same matrix $\tilde{\mathbf{K}}_{\mathrm{m}}$ is inverted to solve the meso-scale BVP (B.3) and to evaluate the apparent tensor (30). As a result the evaluation of the apparent tensor is achieved at negligible cost contrarily to other condensation method, which is of particular interest when stochastic homogenization is performed.

\section{References}

[1] R. Ghanem, P. Spanos, Stochastic Finite Elements: A Spectral Approach, Springer Verlag, 1991. 
[2] O. Lemaitre, O. Knio, Spectral methods for uncertainty quantification with applications to computational fluid dynamics, Springer, 2010.

[3] G. Stefanou, The stochastic finite element method: Past, present and future, Computer Methods in Applied Mechanics and Engineering 198 (912) (2009) 1031 - 1051, ISSN 0045-7825, doi: http://dx.doi.org/10.1016/j.cma.2008.11.007.

[4] K. Alzebdeh, M. Ostoja-Starzewski, Micromechanically based stochastic finite elements: length scales and anisotropy, Probabilistic Engineering Mechanics 11 (4) (1996) 205 - 214, ISSN 0266-8920, doi:http://dx.doi.org/10.1016/0266-8920(96)00015-X, third International Stochastic Structural Dynamics Conference.

[5] M. Ostoja-Starzewski, X. Wang, Stochastic finite elements as a bridge between random material microstructure and global response, Computer Methods in Applied Mechanics and Engineering 168 (14) (1999) 35 - 49, ISSN 0045-7825, doi:http://dx.doi.org/10.1016/S0045-7825(98)00105-4.

[6] L. Mehrez, D. Moens, D. Vandepitte, Stochastic identification of composite material properties from limited experimental databases, part I: Experimental database construction, Mechanical Systems and Signal Processing 27 (2012) 471 - 483, ISSN 0888-3270, doi:http://dx.doi.org/10.1016/j.ymssp.2011.09.004, URL http://www.sciencedirect.com/science/article/pii/S0888327011003724.

[7] L. Mehrez, A. Doostan, D. Moens, D. Vandepitte, Stochastic identification of composite material properties from limited experimental databases, Part II: Uncertainty modelling, Mechanical Systems and Signal Processing 27 (2012) 484 - 498, ISSN 0888-3270, doi:http://dx.doi.org/10.1016/j.ymssp.2011.09.001, URL http://www. sciencedirect.com/science/article/pii/S0888327011003694.

[8] A. Vanaerschot, B. N. Cox, S. V. Lomov, D. Vandepitte, Stochastic framework for quantifying the geometrical variability of laminated textile composites using micro-computed tomography, Composites Part A: Applied Science and Manufacturing 44 (2013) 122 - 131, ISSN 1359-835X, doi:http://dx.doi.org/10.1016/j.compositesa.2012.08.020, URL http://www. sciencedirect.com/science/article/pii/S1359835X12002606.

[9] M. Blacklock, H. Bale, M. Begley, B. Cox, Generating virtual textile composite specimens using statistical data from micro-computed tomography: 1D tow representations for the Binary Model, Journal of the Mechanics and Physics of Solids 60 (3) (2012) 451 - 470, ISSN 0022-5096, doi:http://dx.doi.org/10.1016/j.jmps.2011.11.010, URL http://www.sciencedirect.com/science/article/pii/S0022509611002225. 
[10] D. Tal, J. Fish, Generating a statistically equivalent representative volume element with discrete defects, Composite Structures 153 (2016) 791 - 803, ISSN 0263-8223, doi:http://dx.doi.org/10.1016/j.compstruct.2016.06.077, URL http://www. sciencedirect.com/science/article/pii/S0263822316310790.

[11] M. N. Rossol, T. Fast, D. B. Marshall, B. N. Cox, F. W. Zok, Characterizing In-Plane Geometrical Variability in Textile Ceramic Composites, Journal of the American Ceramic Society 98 (1) (2015) 205-213, ISSN 1551-2916, doi:10.1111/jace.13275, URL http://dx.doi.org/10.1111/jace.13275.

[12] T. Vaughan, C. McCarthy, A combined experimentalnumerical approach for generating statistically equivalent fibre distributions for high strength laminated composite materials, Composites Science and Technology 70 (2) (2010) 291 - 297, ISSN 02663538, doi:http://dx.doi.org/10.1016/j.compscitech.2009.10.020, URL http://www.sciencedirect.com/science/article/pii/S0266353809003832.

[13] M. Hojo, M. Mizuno, T. Hobbiebrunken, T. Adachi, M. Tanaka, S. K. Ha, Effect of fiber array irregularities on microscopic interfacial normal stress states of transversely loaded UD-CFRP from viewpoint of failure initiation, Composites Science and Technology 69 (11) (2009) 1726 - 1734, ISSN 0266-3538, doi:http://dx.doi.org/10.1016/j.compscitech.2008.08.032, URL http://www.sciencedirect.com/science/article/pii/S0266353808003060, experimental Techniques and Design in Composite Materials (ETDCM8) with Regular Papers.

[14] A. Vanaerschot, B. N. Cox, S. V. Lomov, D. Vandepitte, Stochastic multi-scale modelling of textile composites based on internal geometry variability, Computers \& Structures 122 (2013) 55 - 64, ISSN 0045-7949, doi:http://dx.doi.org/10.1016/j.compstruc.2012.10.026, URL http://www.sciencedirect.com/science/article/pii/S0045794912002647, computational Fluid and Solid Mechanics 2013.

[15] A. Melro, P. Camanho, S. Pinho, Generation of random distribution of fibres in long-fibre reinforced composites, Composites Science and Technology 68 (9) (2008) 2092 - 2102, ISSN 02663538, doi:http://dx.doi.org/10.1016/j.compscitech.2008.03.013, URL http://www.sciencedirect.com/science/article/pii/S0266353808001048.

[16] A. Gupta, A. Cecen, S. Goyal, A. K. Singh, S. R. Kalidindi, Structureproperty linkages using a data science approach: Application to a non-metallic inclusion/steel composite system, Acta Materialia 91 (2015) 239 - 254, ISSN 13596454, doi:http://dx.doi.org/10.1016/j.actamat.2015.02.045, URL http://www. sciencedirect.com/science/article/pii/S1359645415001603.

[17] D. Charmpis, G. Schuller, M. Pellissetti, The need for linking micromechanics of materials with stochastic finite elements: A challenge for materials 
science, Computational Materials Science 41 (1) (2007) 27 - 37, ISSN 0927-0256, doi:http://dx.doi.org/10.1016/j.commatsci.2007.02.014, URL http://www.sciencedirect.com/science/article/pii/s0927025607000602.

[18] P. Kanouté, D. P. Boso, J. L. Chaboche, B. A. Schrefler, Multiscale Methods for Composites: A Review, Archives of Computational Methods in Engineering 16 (1) (2009) 31-75, ISSN 1886-1784, doi:10.1007/s11831-0089028-8, URL https://doi.org/10.1007/s11831-008-9028-8.

[19] L. Noels, L. Wu, L. Adam, J. Seyfarth, G. Soni, J. Segurado, G. Laschet, G. Chen, M. Lesueur, M. Lobos, T. Bhlke, T. Reiter, S. Oberpeilsteiner, D. Salaberger, D. Weichert, C. Broeckmann, Effective Properties, in: Handbook of Software Solutions for ICME, Wiley-VCH Verlag GmbH \& Co. KGaA, ISBN 9783527693566, 433-485, doi:10.1002/9783527693566.ch6, URL http://dx.doi.org/10.1002/9783527693566.ch6, 2016.

[20] F. Feyel, Multiscale FE2 elastoviscoplastic analysis of composite structures, Computational Materials Science 16 (1) (1999) 344 - 354, ISSN 0927-0256, doi:http://dx.doi.org/10.1016/S0927-0256(99)00077-4, URL http://www.sciencedirect.com/science/article/pii/S0927025699000774.

[21] J. Michel, H. Moulinec, P. Suquet, Effective properties of composite materials with periodic microstructure: a computational approach, Computer Methods in Applied Mechanics and Engineering 172 (1) (1999) $109 \quad-\quad 143$, ISSN 00457825, doi:http://dx.doi.org/10.1016/S0045-7825(98)00227-8, URL http://www.sciencedirect.com/science/article/pii/S0045782598002278.

[22] K. Terada, M. Hori, T. Kyoya, N. Kikuchi, Simulation of the multi-scale convergence in computational homogenization approaches, International Journal of Solids and Structures 37 (16) (2000) 2285 - 2311, ISSN 0020-7683, doi:http://dx.doi.org/10.1016/S0020-7683(98)00341-2, URL http://www.sciencedirect.com/science/article/pii/S0020768398003412.

[23] V. Kouznetsova, W. A. M. Brekelmans, F. P. T. Baaijens, An approach to micro-macro modeling of heterogeneous materials, Computational Mechanics 27 (1) (2001) 37-48, ISSN 1432-0924, doi:10.1007/s004660000212, URL https://doi.org/10.1007/s004660000212.

[24] C. Miehe, Strain-driven homogenization of inelastic microstructures and composites based on an incremental variational formulation, International Journal for Numerical Methods in Engineering 55 (11) (2002) 1285-1322, ISSN 1097-0207, doi:10.1002/nme.515, URL http://dx.doi.org/10.1002/nme.515.

[25] V. Gusella, F. Cluni, Random field and homogenization for masonry with nonperiodic microstructure, Journal of Mechanics of Materials and Structures 1 (2) (2006) 357-386, ISSN 1559-3959, doi:10.2140/jomms.2006.1.357. 
[26] S. Mariani, R. Martini, A. Ghisi, A. Corigliano, M. Beghi, Overall elastic properties of polysilicon films: A statistical investigation of the effects of polycrystal morphology, International Journal for Multiscale Computational Engineering 9 (3) (2011) 327-346.

[27] D. Savvas, G. Stefanou, M. Papadrakakis, Determination of RVE size for random composites with local volume fraction variation, Computer Methods in Applied Mechanics and Engineering 305 (2016) 340 - 358, ISSN 0045-7825, doi:http://dx.doi.org/10.1016/j.cma.2016.03.002, URL http://www.sciencedirect.com/science/article/pii/S0045782516300822.

[28] X. Yin, W. Chen, A. To, C. McVeigh, W. Liu, Statistical volume element method for predicting microstructureconstitutive property relations, Computer Methods in Applied Mechanics and Engineering 197 (43 - 44) (2008) 3516 - 3529, ISSN 0045-7825, doi: http://dx.doi.org/10.1016/j.cma.2008.01.008, stochastic Modeling of Multiscale and Multiphysics Problems.

[29] A. Liebscher, C. Proppe, C. Redenbach, D. Schwarzer, Uncertainty quantification for metal foam structures by means of image analysis, Probabilistic Engineering Mechanics 28 (2012) 143 - 151, ISSN 02668920, doi:http://dx.doi.org/10.1016/j.probengmech.2011.08.015, URL http://www. sciencedirect.com/science/article/pii/S0266892011000701, computational Stochastic Mechanics $\{\mathrm{CSM} 6\}$.

[30] G. Stefanou, D. Savvas, M. Papadrakakis, Stochastic finite element analysis of composite structures based on material microstructure, Composite Structures 132 (2015) 384 - 392, ISSN 02638223, doi:http://dx.doi.org/10.1016/j.compstruct.2015.05.044, URL http://www. sciencedirect.com/science/article/pii/S0263822315004183.

[31] G. Stefanou, D. Savvas, M. Papadrakakis, Stochastic finite element analysis of composite structures based on mesoscale random fields of material properties, Computer Methods in Applied Mechanics and Engineering (2017) -ISSN 0045-7825, doi:https://doi.org/10.1016/j.cma.2017.08.002, URL http://www.sciencedirect.com/science/article/pii/S0045782517305868.

[32] V. Lucas, J.-C. Golinval, S. Paquay, V.-D. Nguyen, L. Noels, L. Wu, A stochastic computational multiscale approach; Application to MEMS resonators, Computer Methods in Applied Mechanics and Engineering 294 (2015) 141 - 167, ISSN 0045-7825, doi:http://dx.doi.org/10.1016/j.cma.2015.05.019, URL http://www.sciencedirect.com/science/article/pii/S0045782515001929.

[33] L. Wu, V. Lucas, V.-D. Nguyen, J.-C. Golinval, S. Paquay, L. Noels, A stochastic multi-scale approach for the modeling of thermoelastic damping in micro-resonators, Computer Methods in Applied Mechanics and Engineering 310 (2016) 802 - 839, ISSN 
0045-7825, doi:http://dx.doi.org/10.1016/j.cma.2016.07.042, URL http://www.sciencedirect.com/science/article/pii/S0045782516303930.

[34] P. Trovalusci, M. L. De Bellis, M. Ostoja-Starzewski, A. Murrali, Particulate random composites homogenized as micropolar materials, Meccanica 49 (11) (2014) 2719-2727, ISSN 1572-9648, doi:10.1007/s11012-014-0031-x, URL https://doi.org/10.1007/s11012-014-0031-x.

[35] P. Trovalusci, M. Ostoja-Starzewski, M. L. De Bellis, A. Murrali, Scaledependent homogenization of random composites as micropolar continua, European Journal of Mechanics - A/Solids 49 (0) (2015) 396 - 407, ISSN 0997-7538, doi:http://dx.doi.org/10.1016/j.euromechsol.2014.08.010.

[36] V. Lucas, J.-C. Golinval, R. C. Voicu, M. Danila, R. Gavrila, R. Mller, A. Dinescu, L. Noels, L. Wu, Propagation of material and surface profile uncertainties on MEMS micro-resonators using a stochastic second-order computational multi-scale approach, International Journal for Numerical Methods in Engineering 111 (1) (2017) 26-68, ISSN 1097-0207, doi:10.1002/nme.5452, URL http://dx.doi.org/10.1002/nme.5452, nme. 5452 .

[37] S. C. Baxter, L. L. Graham, Characterization of Random Composites Using Moving-Window Technique, Journal of Engineering Mechanics 126 (4) (2000) 389-397, doi:10.1061/(ASCE)0733-9399(2000)126:4(389), URL http: //ascelibrary.org/doi/abs/10. 1061/\%28ASCE $\% 290733-9399 \% 282000 \% 29126 \% 3$ A4\%28389\%29.

[38] X. Yin, S. Lee, W. Chen, W. K. Liu, M. F. Horstemeyer, Efficient Random Field Uncertainty Propagation in Design Using Multiscale Analysis, Journal of Mechanical Design 131 (2) (2009) 021006, ISSN 1050-0472, doi: http://dx.doi.org/10.1115/1.3042159.

[39] J. Guilleminot, A. Noshadravan, C. Soize, R. Ghanem, A probabilistic model for bounded elasticity tensor random fields with application to polycrystalline microstructures, Computer Methods in Applied Mechanics and Engineering 200 (17 - 20) (2011) 1637 - 1648, ISSN 0045-7825, doi:http://dx.doi.org/10.1016/j.cma.2011.01.016.

[40] A. Noshadravan, R. Ghanem, J. Guilleminot, I. Atodaria, P. Peralta, Validation of a probabilistic model for mesoscale elasticity tensor or random polycrystals, International Journal for Uncertainty Quantification 3 (1) (2013) 73-100, ISSN 2152-5080.

[41] M. Shinozuka, Simulation of Multivariate and Multidimensional Random Processes, The Journal of the Acoustical Society of America 49 (1B) (1971) 357-368, doi:http://dx.doi.org/10.1121/1.1912338.

[42] R. Popescu, G. Deodatis, J. Prevost, Simulation of homogeneous nonGaussian stochastic vector fields, Probabilistic Engineering Mechanics 13 (1) (1998) 1 - 13, ISSN 0266-8920, doi:10.1016/S0266-8920(97)000015. 
[43] F. Yamazaki, M. Shinozuka, Digital generation of non-Gaussian stochastic fields, Journal of Engineering Mechanics 114 (7) (1988) 1183-1197.

[44] G. Deodatis, R. C. Micaletti, Simulation of highly skewed non-Gaussian stochastic processes, Journal of engineering mechanics 127 (12) (2001) $1284-1295$.

[45] A. Maligno, N. Warrior, A. Long, Effects of inter-fibre spacing on damage evolution in unidirectional (UD) fibre-reinforced composites, European Journal of Mechanics - A/Solids 28 (4) (2009) 768 - 776, ISSN 0997-7538, doi:http://dx.doi.org/10.1016/j.euromechsol.2008.10.009, URL http://www.sciencedirect.com/science/article/pii/S0997753808001113.

[46] T. Hobbiebrunken, M. Hojo, K. Jin, S. Ha, Influence of non-uniform fiber arrangement on microscopic stress and failure initiation in thermally and transversely loaded CF/epoxy laminated composites, Composites Science and Technology 68 (15) (2008) 3107 - 3113, ISSN 0266-3538, doi:http://dx.doi.org/10.1016/j.compscitech.2008.07.006, URL http://www. sciencedirect.com/science/article/pii/S0266353808002637.

[47] L. Wu, L. Noels, L. Adam, I. Doghri, An implicit-gradientenhanced incremental-secant mean-field homogenization scheme for elasto-plastic composites with damage, International Journal of Solids and Structures 50 (24) (2013) 3843 - 3860, ISSN 0020-7683, doi: 10.1016/j.ijsolstr.2013.07.022.

[48] L. Wu, F. Sket, J. Molina-Aldareguia, A. Makradi, L. Adam, I. Doghri, L. Noels, A study of composite laminates failure using an anisotropic gradient-enhanced damage mean-field homogenization model, Composite Structures 126 (2015) 246 - 264, ISSN 02638223, doi:http://dx.doi.org/10.1016/j.compstruct.2015.02.070, URL http://www.sciencedirect.com/science/article/pii/S0263822315001580.

[49] M. Bansal, I. Singh, B. Mishra, S. Kamal, K. I.A., A stochastic XFEM model for the tensile strength prediction of heterogeneous graphite based on microstructural observations, Journal of Nuclear Materials 487 (Supplement C) (2017) 143 - 157, ISSN 0022-3115, doi:https://doi.org/10.1016/j.jnucmat.2016.12.045, URL http://www.sciencedirect.com/science/article/pii/S0022311516306973.

[50] M. Bansal, I. V. Singh, B. Mishra, K. Sharma, I. Khan, A two-scale stochastic framework for predicting failure strength probability of heterogeneous materials, Composite Structures 179 (Supplement C) (2017) 294 - 325, ISSN 02638223, doi:https://doi.org/10.1016/j.compstruct.2017.07.044, URL http://www. sciencedirect.com/science/article/pii/S0263822317315088.

[51] M. Olave, A. Vanaerschot, S. V. Lomov, D. Vandepitte, Internal geometry variability of two woven composites and related variability of the 
stiffness, Polymer Composites 33 (8) (2012) 1335-1350, ISSN 1548-0569, doi:10.1002/pc.22260, URL http://dx.doi.org/10.1002/pc. 22260.

[52] J. Illian, A. Penttinen, H. Stoyan, D. Stoyan, Statistical analysis and modelling of spatial point patterns, vol. 70, John Wiley \& Sons, 2008.

[53] G. J. Szkely, M. L. Rizzo, N. K. Bakirov, Measuring and testing dependence by correlation of distances, Ann. Statist. 35 (6) (2007) 2769-2794, doi:10.1214/009053607000000505, URL http://dx.doi.org/10.1214/009053607000000505.

[54] J. C. Strelen, F. Nassaj, Analysis and Generation of Random Vectors with Copulas, in: Proceedings of the 39th Conference on Winter Simulation: 40 Years! The Best is Yet to Come, WSC '07, IEEE Press, Piscataway, NJ, USA, ISBN 1-4244-1306-0, 488-496, URL http://dl.acm.org/citation. cfm?id=1351542.1351639, 2007.

[55] S. H. Reza Sanei, E. J. Barsotti, D. Leonhardt, R. S. I. Fertig, Characterization, synthetic generation, and statistical equivalence of composite microstructures, Journal of Composite Materials 51 (13) (2017) 1817-1829, doi:10.1177/0021998316662133.

[56] C. Miehe, A. Koch, Computational micro-to-macro transitions of discretized microstructures undergoing small strains, Archive of Applied Mechanics 72 (4-5) (2002) 300-317, ISSN 0939-1533, doi:10.1007/s00419-0020212-2.

[57] M. Ainsworth, Essential boundary conditions and multi-point constraints in finite element analysis, Computer Methods in Applied Mechanics and Engineering 190 (48) (2001) 6323 - 6339, ISSN 00457825, doi:http://dx.doi.org/10.1016/S0045-7825(01)00236-5, URL http://www.sciencedirect.com/science/article/pii/S0045782501002365.

[58] V.-D. Nguyen, L. Wu, L. Noels, Unified treatment of microscopic boundary conditions and efficient algorithms for estimating tangent operators of the homogenized behavior in the computational homogenization method, Computational Mechanics 59 (3) (2017) 483-505, ISSN 1432-0924, doi:10.1007/s00466-016-1358-z, URL https://doi.org/10.1007/s00466-016-1358-z.

[59] D. Peric, E. A. de Souza Neto, R. A. Feijóo, M. Partovi, A. J. C. Molina, On micro-to-macro transitions for multi-scale analysis of non-linear heterogeneous materials: unified variational basis and finite element implementation, International Journal for Numerical Methods in Engineering 87 (2010) 149 - 170, ISSN 1097-0207, URL http://dx. doi.org/10.1002/nme. 3014.

[60] J. Schröder, M. Labusch, M.-A. Keip, Algorithmic two-scale transition for magneto-electro-mechanically coupled problems: FE2scheme: Localization and homogenization, Computer Methods 
in Applied Mechanics and Engineering 32 (2016) 253-280, ISSN 0045-7825, doi:http://dx.doi.org/10.1016/j.cma.2015.10.005, URL http://www.sciencedirect.com/science/article/pii/S0045782515003242.

[61] V.-D. Nguyen, E. Béchet, C. Geuzaine, L. Noels, Imposing periodic boundary condition on arbitrary meshes by polynomial interpolation, Computational Materials Science 55 (0) (2012) 390 - 406, ISSN 0927-0256, doi:10.1016/j.commatsci.2011.10.017, URL http://www.sciencedirect.com/science/article/pii/S0927025611005866.

[62] M. Shinozuka, G. Deodatis, Response Variability Of Stochastic Finite Element Systems, Journal of Engineering Mechanics 114 (3) (1988) 499-519, doi:10.1061/(ASCE)0733-9399(1988)114:3(499).

[63] C. Soize, R. Ghanem, Data-driven probability concentration and sampling on manifold, Journal of Computational Physics 321 (2016) 242 - 258, ISSN 0021-9991, doi:http://dx.doi.org/10.1016/j.jcp.2016.05.044, URL http://www.sciencedirect.com/science/article/pii/S0021999116301899.

[64] D. Pivovarov, P. Steinmann, Modified SFEM for computational homogenization of heterogeneous materials with microstructural geometric uncertainties, Computational Mechanics 57 (1) (2016) 123-147.

[65] J. Fish, W. Wu, A nonintrusive stochastic multiscale solver, International Journal for Numerical Methods in Engineering 88 (9) (2011) 862-879, ISSN 1097-0207, doi:10.1002/nme.3201.

[66] A. Clément, C. Soize, J. Yvonnet, Computational nonlinear stochastic homogenization using a nonconcurrent multiscale approach for hyperelastic heterogeneous microstructures analysis, International Journal for Numerical Methods in Engineering 91 (8) (2012) 799-824, ISSN 1097-0207, doi: $10.1002 /$ nme.4293.

[67] J. Yvonnet, E. Monteiro, Q.-C. He, Computational homogenization method and reduced database model for hyperelastic heterogeneous structures, International Journal for Multiscale Computational Engineering 11 (3) (2013) 201-225, ISSN 1543-1649.

[68] M. Bessa, R. Bostanabad, Z. Liu, A. Hu, D. W. Apley, C. Brinson, W. Chen, W. Liu, A framework for data-driven analysis of materials under uncertainty: Countering the curse of dimensionality, Computer Methods in Applied Mechanics and Engineering 320 (2017) 633 - 667, ISSN 0045-7825, doi:http://dx.doi.org/10.1016/j.cma.2017.03.037, URL http://www. sciencedirect.com/science/article/pii/S0045782516314803. 